\title{
Long-Term Efficacy and Safety of Pemafibrate, a Novel Selective Peroxisome Proliferator-Activated Receptor- $\alpha$ Modulator (SPPARM $\alpha$ ), in Dyslipidemic Patients with Renal Impairment
}

\author{
Koutaro Yokote ${ }^{1,2, *}$, Shizuya Yamashita ${ }^{3,4}$, Hidenori Arai ${ }^{5}$, Eiichi Araki ${ }^{6}$, Hideki Suganami ${ }^{7}$, \\ Shun Ishibashi ${ }^{8}$ and on Behalf of the K-877 Study Group \\ 1 Department of Diabetes, Metabolism and Endocrinology, Chiba University Hospital, Chiba 260-8670, Japan \\ 2 Department of Endocrinology, Hematology and Gerontology, \\ Chiba University Graduate School of Medicine, Chiba 260-8670, Japan \\ 3 Department of Community Medicine and Department of Cardiovascular Medicine, \\ Osaka University Graduate School of Medicine, Osaka 565-0871, Japan; shizu@imed2.med.osaka-u.ac.jp \\ 4 Rinku General Medical Center, Osaka 598-8577, Japan \\ 5 National Center for Geriatrics and Gerontology, Aichi 474-8511, Japan; harai@ncgg.go.jp \\ 6 Department of Metabolic Medicine, Faculty of Life Sciences, Kumamoto University, Kumamoto 860-8556, \\ Japan; earaki@gpo.kumamoto-u.ac.jp \\ 7 Clinical Data Science Department, Kowa Company, Ltd., Tokyo 103-8433, Japan; suganami@kowa.co.jp \\ 8 Division of Endocrinology and Metabolism, Department of Medicine, Jichi Medical University, \\ Tochigi 329-0498, Japan; ishibash@jichi.ac.jp \\ * Correspondence: kyokote@faculty.chiba-u.jp; Tel.: +81-43-226-2089
}

Received: 9 January 2019; Accepted: 3 February 2019; Published: 6 February 2019

\begin{abstract}
Pemafibrate (K-877) is a novel selective peroxisome proliferator-activated receptor- $\alpha$ modulator (SPPARM $\alpha$ ) with a favorable benefit-risk balance. Previous clinical trials of pemafibrate used stringent exclusion criteria related to renal functions. Therefore, we investigated its safety and efficacy in a broader range of patients, including those with chronic kidney disease (CKD). In this multicenter, single-arm, open-label, phase III trial, $0.2-0.4 \mathrm{mg} /$ day pemafibrate was administered for 52 weeks to 189 patients with hypertriglyceridemia and an estimated glomerular filtration rate (eGFR) $\geq 45 \mathrm{~mL} / \mathrm{min} / 1.73 \mathrm{~m}^{2}$ on statin or regardless of eGFR when statin was not administered. Post-hoc analyses were performed on subgroups stratified by baseline eGFR. Triglyceride levels decreased by $45.9 \%$ at week 52 (last-observation-carried-forward). These reductions were not correlated with baseline eGFR. The eGFR $<30 \mathrm{~mL} / \mathrm{min} / 1.73 \mathrm{~m}^{2}$ subgroup showed the greatest reduction in chylomicron, very low-density lipoprotein, small low-density lipoprotein cholesterol levels, and an increase in high-density lipoprotein cholesterol levels. The incidences of adverse events and adverse drug reactions were $82.0 \%$ and $31.7 \%$, respectively, and these were not associated with baseline eGFR. In CKD patients, pemafibrate blood concentrations were not elevated. Pemafibrate showed a good safety profile and efficacy in correcting lipid abnormalities in a broad range of patients, including those with CKD.
\end{abstract}

Keywords: high-density lipoprotein cholesterol; K-877; pemafibrate; renal dysfunction; safety; selective PPAR $\alpha$ modulator; triglyceride

\section{Introduction}

Atherosclerotic cardiovascular disease (ASCVD) is a leading cause of death [1,2]. Reducing low-density lipoprotein (LDL) cholesterol (LDL-C) levels is an established pharmacotherapy for 
ASCVD prevention, and statins are preferred for this purpose. Residual lipid abnormalities, such as elevated triglyceride (TG) and/or reduced high-density lipoprotein (HDL)-cholesterol (HDL-C) levels, are secondary targets [3,4].

Reduced renal function is associated with elevated risks of cardiovascular disease (CVD) and death [5,6]. Atherogenic dyslipidemia, characterized by increased TG-rich lipoproteins and decreased HDL-C levels, is frequently observed in patients with reduced renal function, which itself is associated with CVD risk [7]. Although atherogenic dyslipidemia can be ameliorated with peroxisome proliferator-activated receptor $\alpha(\operatorname{PPAR} \alpha)$ agonists [8], clinical trials have shown that these agents increase serum creatinine levels [9-14]. This adverse reaction may require a dose reduction or treatment discontinuation in patients with reduced renal function [15-17]. Additionally, statins and PPAR $\alpha$ activators may increase the risk of developing myopathy and rhabdomyolysis, which is greater in patients with chronic kidney disease (CKD) $[15,18,19]$. Therefore, the concomitant use of these drugs should be avoided in these patients, whereas the risks vary with different fibrates and statins used in combination [20].

Pemafibrate (K-877) is a novel selective PPAR $\alpha$ modulator (SPPARM $\alpha$ ). It was designed to have a favorable benefit-risk balance and is now approved for the treatment of hyperlipidemia in Japan [21-29]. Compared to other PPAR $\alpha$ agonists, pemafibrate does not significantly increase, and may even decrease, alanine aminotransferase (ALT) or $\gamma$-glutamyltransferase $(\gamma$-GT) levels [22,24,26]. Moreover, unlike many other PPAR $\alpha$ agonists, pemafibrate is principally excreted via the liver $[15,27,30]$ and exposure to pemafibrate is not dependent on the severity of renal dysfunction $[27,31]$.

The present study is a long-term (52-week) phase III trial of pemafibrate involving patients with an unprecedented broad range of characteristics. Unlike previous trials, this study used less stringent exclusion criteria related to hepatic and renal functions. The main data and results of the post-hoc subgroup analyses on the relative efficacy and safety of pemafibrate with respect to the baseline estimated glomerular filtration rate (eGFR) are presented. The latter analyses were performed because a significant number of patients with renal impairment were enrolled in this study.

\section{Results}

\subsection{Patients}

Of the 295 patients who provided written informed consent, 189 received pemafibrate, 105 dropped out during the screening period, and one discontinued use due to an adverse event (AE) occurring before pemafibrate treatment (Figure 1). The baseline patient characteristics are presented in Table 1. The age of the patients was $57.8 \pm 10.5$ years, and $77.8 \%$ were men. Their body mass index (BMI) was $26.0 \pm 3.5 \mathrm{~kg} / \mathrm{m}^{2}$, and $37.0 \%$ of the patients had type 2 diabetes mellitus, $53.4 \%$ had hypertension, $74.1 \%$ had fatty liver, and $57.1 \%$ received concomitant statin treatment. TG, $\mathrm{HDL}-\mathrm{C}$, and $\mathrm{HbA} 1 \mathrm{c}$ levels were $2.82 \pm 0.88 \mathrm{mmol} / \mathrm{L}, 1.18 \pm 0.27 \mathrm{mmol} / \mathrm{L}$, and $6.3 \pm 0.9 \%$, respectively. In the baseline eGFR subgroups, 21, 123, 34, and eight patients were in the G1, G2, G3a-G3b, and G4-G5 groups, respectively (see "Subjects and Methods" section). Three other patients underwent hemodialysis. The mean age of the patients in the G3a-G3b and G4-G5 subgroups was 12 years older than that of the patients in the G1 group. There were relatively higher rates of hypertension among the patients in the lower eGFR categories. There was no clear correlation between baseline TG or LDL-C and baseline eGFR. The lowest baseline HDL-C, $0.87 \pm 0.22 \mathrm{mmol} / \mathrm{L}$, was found in the G4-G5 subgroup. 


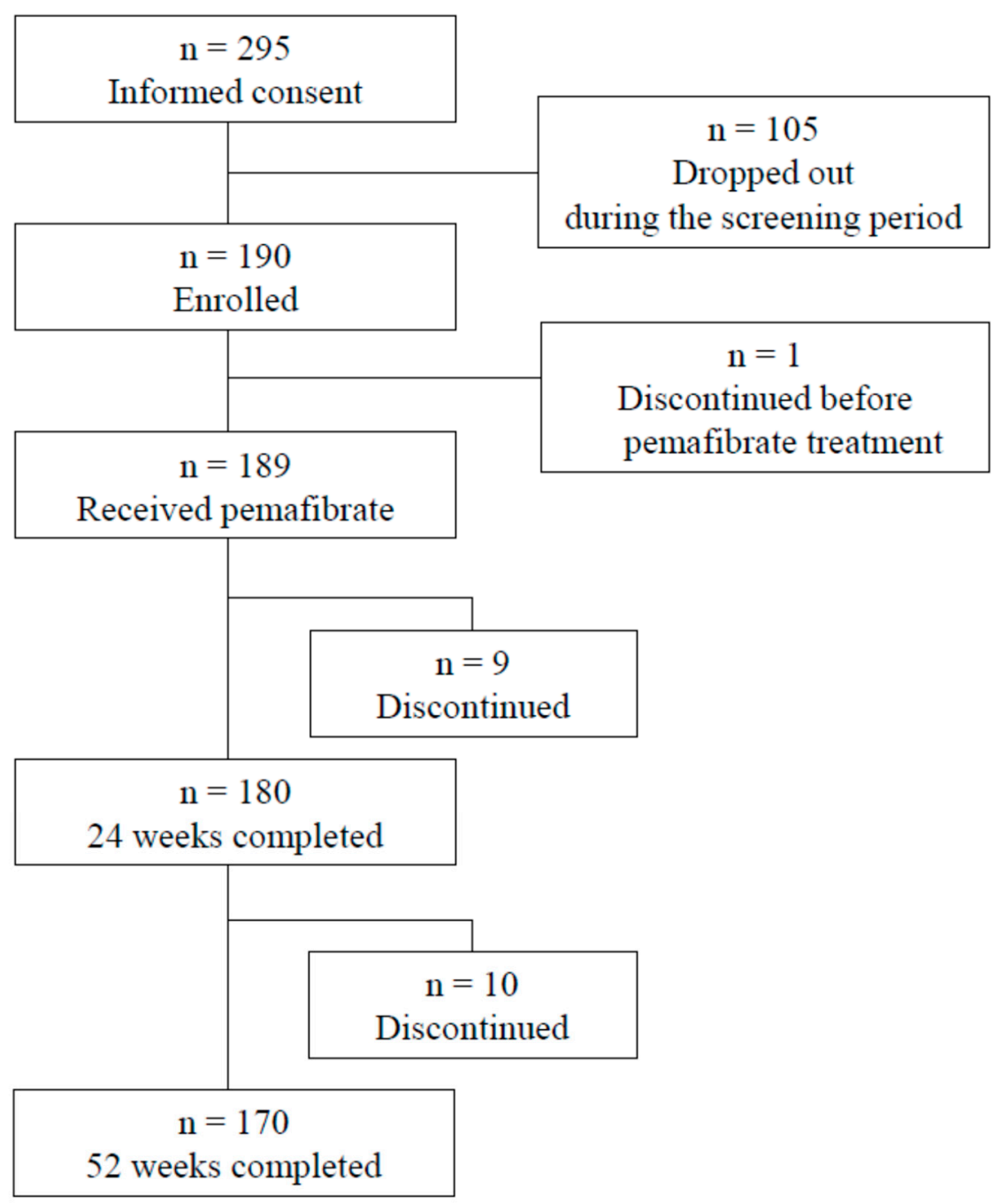

Figure 1. Flow chart of included and excluded patients. 
Table 1. Characteristics of patients at baseline.

\begin{tabular}{|c|c|c|c|c|c|c|}
\hline \multirow{2}{*}{ Parameter } & \multirow{2}{*}{ All Participants } & \multicolumn{4}{|c|}{ Baseline eGFR Category } & \multirow{2}{*}{ Hemodialysis } \\
\hline & & G1 & G2 & G3a-G3b & G4-G5 & \\
\hline$n$ & 189 & 21 & 123 & 34 & 8 & 3 \\
\hline Age (years) & $57.8(10.5)$ & $52.6(9.8)$ & $55.9(9.3)$ & $65.5(9.7)$ & $64.6(12.8)$ & $67.7(12.5)$ \\
\hline Sex, Men & $147(77.8)$ & $16(76.2)$ & $98(79.7)$ & $25(73.5)$ & 7 (87.5) & $1(33.3)$ \\
\hline $\operatorname{BMI}\left(\mathrm{kg} / \mathrm{m}^{2}\right)$ & $26.0(3.5)$ & $26.6(4.6)$ & $26.1(3.4)$ & $25.8(2.5)$ & $26.0(2.3)$ & $23.4(7.3)$ \\
\hline Type 2 diabetes & $70(37.0)$ & $10(47.6)$ & $39(31.7)$ & $16(47.1)$ & $4(50.0)$ & $1(33.3)$ \\
\hline Hypertension & $101(53.4)$ & $8(38.1)$ & $57(46.3)$ & $26(76.5)$ & $7(87.5)$ & $3(100)$ \\
\hline Fatty liver & $140(74.1)$ & $19(90.5)$ & $91(74.0)$ & $26(76.5)$ & $4(50.0)$ & 0 \\
\hline Use of a statin & $108(57.1)$ & $11(52.4)$ & $77(62.6)$ & $18(52.9)$ & $2(25.0)$ & 0 \\
\hline TG (mmol/L) & $2.82(0.88)$ & $2.94(1.03)$ & $2.80(0.89)$ & $2.88(0.86)$ & $2.72(0.47)$ & $2.47(0.53)$ \\
\hline HDL-C (mmol/L) & $1.18(0.27)$ & $1.24(0.36)$ & $1.19(0.24)$ & $1.19(0.31)$ & $0.87(0.22)$ & $1.12(0.42)$ \\
\hline LDL-C $(\mathrm{mmol} / \mathrm{L})$ & $3.09(0.82)$ & $3.20(0.88)$ & $3.08(0.78)$ & $2.96(0.72)$ & $3.42(1.18)$ & $3.45(2.09)$ \\
\hline Serum creatinine $(\mathrm{mg} / \mathrm{dL})$ & $1.05(1.00)$ & $0.62(0.10)$ & $0.82(0.12)$ & $1.09(0.25)$ & $3.23(1.30)$ & 7.35 (1.68) \\
\hline $\mathrm{eGFR}\left(\mathrm{mL} / \mathrm{min} / 1.73 \mathrm{~m}^{2}\right)$ & $68.6(20.3)$ & $99.6(8.0)$ & $72.9(8.5)$ & $51.1(7.0)$ & $18.2(6.9)$ & $5.6(1.4)$ \\
\hline $\mathrm{HbA} 1 \mathrm{c}(\%)$ & $6.3(0.9)$ & $6.5(1.1)$ & $6.2(0.8)$ & $6.6(1.0)$ & $6.1(0.4)$ & $6.7(1.9)$ \\
\hline
\end{tabular}

Data are presented as means (SD) for continuous parameters and $n$ (\%) for categorical parameters. Baseline eGFR categories are as follows: G1, eGFR $\geq 90 \mathrm{~mL} / \mathrm{min} / 1.73 \mathrm{~m}{ }^{2} ; \mathrm{G} 2, \mathrm{eGFR} \geq 60$ and $<90 \mathrm{~mL} / \mathrm{min} / 1.73 \mathrm{~m}^{2}$; G3a-G3b, eGFR $\geq 30$ and $<60 \mathrm{~mL} / \mathrm{min} / 1.73 \mathrm{~m}^{2}$; G4-G5, eGFR $<30 \mathrm{~mL} / \mathrm{min} / 1.73 \mathrm{~m}^{2}$. BMI, body mass index; TG, triglyceride; HDL-C, high-density lipoprotein cholesterol; LDL-C, low-density lipoprotein cholesterol; eGFR, estimated glomerular filtration rate; HbA1c, hemoglobin A1c; SD, standard deviation. 


\subsection{Efficacy}

Fasting serum TG levels decreased by $45.9 \%$ from baseline to the last observation $(p<0.001)$ and significantly decreased from baseline over the 52 weeks at each time point $(p<0.001$; Figure 2). During that time, the pemafibrate dose was increased from $0.2 \mathrm{mg} /$ day to $0.4 \mathrm{mg} /$ day in 29 patients. This adjustment further reduced the TG levels in 17 patients (58.6\%). There were no large differences in percent TG reduction either among the prespecified subgroups (Figure 3) or the subgroups categorized by baseline eGFR (Figure 4 and Table 2). The TG reduction in three patients receiving hemodialysis was comparable to that in the total population: Case 1, from 2.62 to $1.39 \mathrm{mmol} / \mathrm{L}(-46.9 \%)$; Case 2, from 3.04 to $1.72 \mathrm{mmol} / \mathrm{L}(-43.4 \%)$; Case 3, from 1.93 to $0.82 \mathrm{mmol} / \mathrm{L}(-57.5 \%)$ during the 52-week treatment.

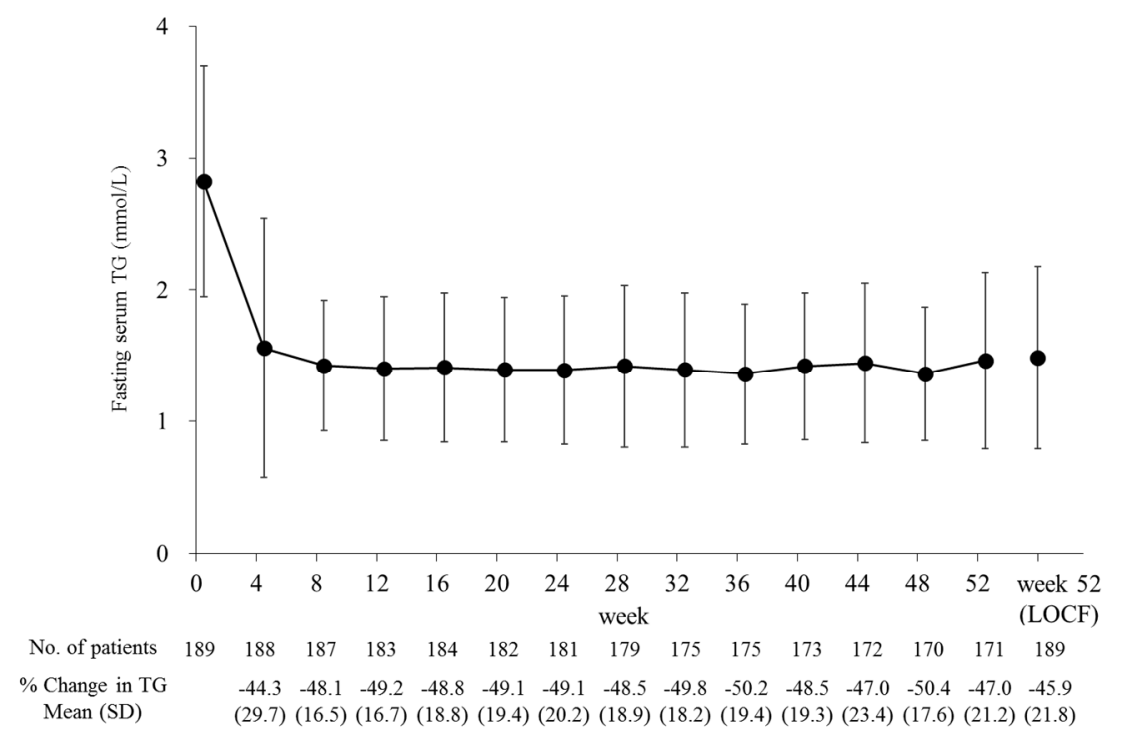

Figure 2. Time course of fasting serum TG levels. Data are presented as means \pm SD. TG, triglyceride; LOCF, last-observation-carried-forward; SD, standard deviation.

\begin{tabular}{|c|c|c|c|c|c|}
\hline & & & aseline TG $(\mathrm{mmol}$ & & \\
\hline Age, years & $<65$ & $(n=132)$ & $2.87(0.91)$ & $\rightarrow$ & $-45.4(-49.4,-41.5)$ \\
\hline & $\geq 65$ & $(n=57)$ & $2.72(0.78)$ & $\rightarrow$ & $-47.1(-52.3,-41.8)$ \\
\hline Sex & Men & $(n=147)$ & $2.93(0.91)$ & $\rightarrow$ & $-44.2(-47.8,-40.5)$ \\
\hline & Women & $(n=42)$ & $2.44(0.61)$ & $\rightarrow$ & $-52.2(-58.0,-46.3)$ \\
\hline BMI, $\mathrm{kg} / \mathrm{m}^{2}$ & $<25$ & $(n=80)$ & $2.93(0.85)$ & $\rightarrow-$ & $-47.2(-52.6,-41.8)$ \\
\hline & $\geq 25$ & $(n=109)$ & $2.74(0.89)$ & & $-45.0(-48.8,-41.2)$ \\
\hline Type 2 DM & Absent & $(n=119)$ & $2.79(0.93)$ & $\rightarrow-$ & $-45.7(-49.8,-41.6)$ \\
\hline & Present & $(n=70)$ & $2.88(0.79)$ & $\rightarrow$ & $-46.3(-51.2,-41.4)$ \\
\hline Mets & Absent & $(n=67)$ & $2.92(1.00)$ & $\rightarrow$ & $-48.6(-54.7,-42.5)$ \\
\hline & Present & $(n=122)$ & $2.77(0.80)$ & - & $-44.5(-48.0,-40.9)$ \\
\hline Any statin & Absent & $(n=81)$ & $2.99(0.98)$ & $\rightarrow-$ & $-45.5(-50.2,-40.8)$ \\
\hline & Present & $(n=108)$ & $2.70(0.77)$ & $\rightarrow-$ & $-46.3(-50.6,-42.0)$ \\
\hline Fatty liver & Absent & $(n=49)$ & $2.92(0.87)$ & $\rightarrow$ & $-51.0(-56.6,-45.5)$ \\
\hline & Present & $(n=140)$ & $2.79(0.88)$ & $\rightarrow$ & $-44.2(-47.9,-40.4)$ \\
\hline Baseline HDL-C, & $<1.04$ & $(n=57)$ & $3.30(1.14)$ & $\rightarrow$ & $-42.7(-48.0,-37.5)$ \\
\hline $\mathrm{mmol} / \mathrm{L}$ & $\geq 1.04$ & $(n=132)$ & $2.62(0.63)$ & - & $-47.3(-51.2,-43.4)$ \\
\hline Timing of & Preprandial & $(n=15)$ & $3.11(0.57)$ & $\longrightarrow$ & $-48.9(-57.6,-40.2)$ \\
\hline administration & Postprandial & $1(n=174)$ & $2.80(0.89)$ & $\rightarrow$ & $-45.7(-49.0,-42.3)$ \\
\hline & & & -8 & $\begin{array}{lll}-60 & -40 & -20 \\
\text { Percent change of } T G\end{array}$ & ) \\
\hline
\end{tabular}

Figure 3. Subgroup analysis for percent change of TG from baseline at week 52 (LOCF). Data of percent change of TG are presented as means (95\% CI), and data of baseline TG are presented as means (SD). TG, triglyceride; LOCF, last-observation-carried-forward; SD, standard deviation; CI, confidence interval; BMI, body mass index; DM, diabetes mellitus; Mets, metabolic syndrome. 


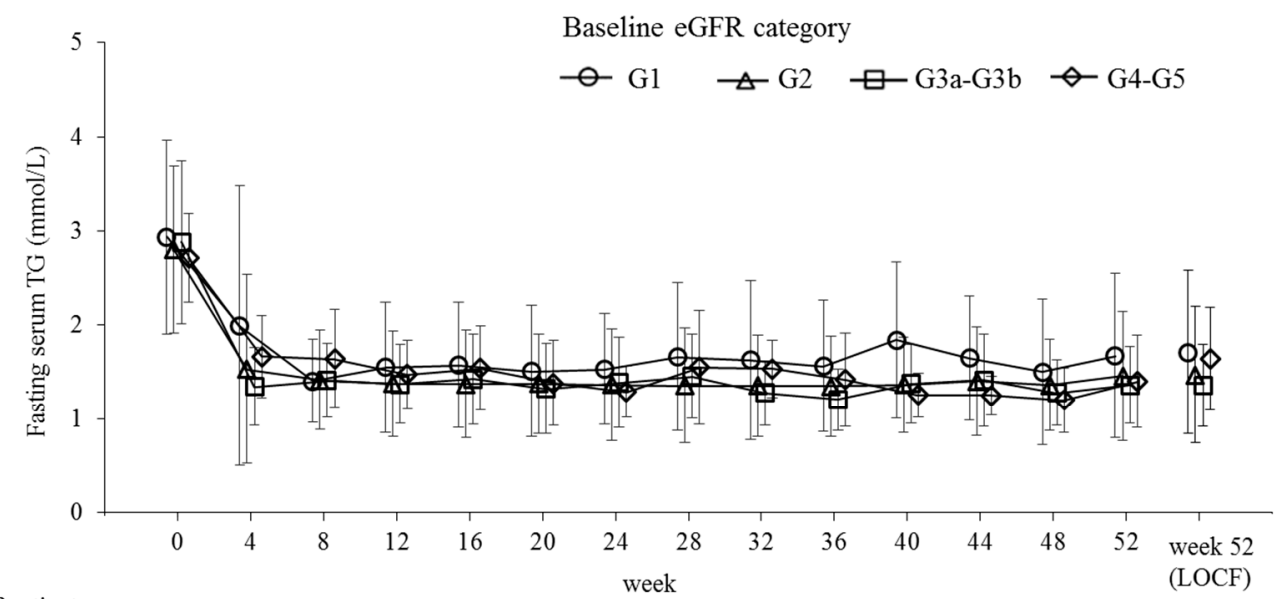

No. of patients

$\begin{array}{cccccccccccccccc}\text { G1 } & 21 & 21 & 21 & 21 & 21 & 21 & 21 & 21 & 21 & 21 & 20 & 19 & 19 & 19 & 21 \\ \text { G2 } & 123 & 123 & 122 & 121 & 121 & 121 & 121 & 121 & 118 & 117 & 116 & 116 & 116 & 116 & 123 \\ \text { G3a-G3b } & 34 & 33 & 33 & 32 & 33 & 32 & 31 & 30 & 29 & 29 & 29 & 29 & 27 & 28 & 34 \\ \text { G4-G5 } & 8 & 8 & 8 & 6 & 6 & 5 & 5 & 4 & 4 & 5 & 5 & 5 & 5 & 5 & 8\end{array}$

Figure 4. Time course of fasting serum TG levels by baseline eGFR category. Baseline eGFR categories are as follows: G1, eGFR $\geq 90 \mathrm{~mL} / \mathrm{min} / 1.73 \mathrm{~m}^{2}$; G2, eGFR $\geq 60$ and $<90 \mathrm{~mL} / \mathrm{min} / 1.73 \mathrm{~m}^{2}$; G3a-G3b, eGFR $\geq 30$ and $<60 \mathrm{~mL} / \mathrm{min} / 1.73 \mathrm{~m}^{2}$; and G4-G5, eGFR $<30 \mathrm{~mL} / \mathrm{min} / 1.73 \mathrm{~m}^{2}$. Data are presented as means \pm standard deviation. TG, triglyceride; eGFR, estimated glomerular filtration rate; LOCF, last-observation-carried-forward.

Pemafibrate treatment significantly increased HDL-C, apolipoprotein (apo) A1, and apoA2 levels, and significantly reduced TG/HDL-C, non-HDL-C, remnant lipoprotein cholesterol (RemL-C), apoB, apoB48, apoC3, and interleukin-1 $\beta$ (IL-1 $\beta$ ) levels (Table 2). There were no significant changes in LDL-C levels. High-performance liquid chromatography (HPLC) analysis revealed that the cholesterol content significantly decreased in TG-rich lipoproteins such as chylomicrons (CMs) and very-low-density lipoproteins (VLDLs), and in small to very small LDL particles. In contrast, the cholesterol content significantly increased in medium to very small HDL particles (Table 3). In subgroups with lower baseline eGFR, there were relatively large increases in apoA1, apoA2, HDL-C, medium HDL-C, and small HDL-C, and large decreases in CM-cholesterol (CM-C), VLDL-cholesterol (VLDL-C), small LDL-C, and very small LDL-C levels (Tables 2 and 3). 
Table 2. Changes from baseline in lipid levels and inflammatory parameters during the 52-week treatment period.

\begin{tabular}{|c|c|c|c|c|c|c|}
\hline \multicolumn{2}{|c|}{ Parameter } & \multirow{2}{*}{ All Participants } & \multicolumn{4}{|c|}{ Baseline eGFR Category } \\
\hline & & & G1 & G2 & G3a-G3b & G4-G5 \\
\hline \multirow{4}{*}{$\mathrm{TG}(\mathrm{mmol} / \mathrm{L})$} & $n$ & 189 & 21 & 123 & 34 & 8 \\
\hline & Baseline & $2.82(0.88)$ & $2.94(1.03)$ & $2.80(0.89)$ & $2.88(0.86)$ & $2.72(0.47)$ \\
\hline & Week 52 (LOCF) & $1.48(0.69)$ & $1.71(0.87)$ & $1.47(0.72)$ & $1.36(0.44)$ & $1.64(0.54)$ \\
\hline & $\%$ Change & $-45.9(21.8)^{* * *}$ & $-41.4(23.0)^{* * *}$ & $-45.8(23.1)^{* * *}$ & $-51.1(15.4)^{* * *}$ & $-37.7(23.6)^{* *}$ \\
\hline \multirow{4}{*}{ HDL-C (mmol/L) } & $n$ & 189 & 21 & 123 & 34 & 8 \\
\hline & Baseline & $1.18(0.27)$ & $1.24(0.36)$ & $1.19(0.24)$ & $1.19(0.31)$ & $0.87(0.22)$ \\
\hline & Week 52 (LOCF) & $1.33(0.34)$ & $1.29(0.44)$ & $1.34(0.32)$ & $1.38(0.35)$ & $1.18(0.37)$ \\
\hline & $\%$ Change & $13.1(17.1)^{* * *}$ & $3.6(16.9)$ & $11.9(16.2)^{* * *}$ & $17.0(13.0)^{* * *}$ & $34.1(23.7)^{* *}$ \\
\hline \multirow{4}{*}{$\begin{array}{c}\text { TG/HDL-C } \\
{[(\mathrm{mmol} / \mathrm{L}) /(\mathrm{mmol} / \mathrm{L})]}\end{array}$} & $n$ & 189 & 21 & 123 & 34 & 8 \\
\hline & Baseline & $2.58(1.22)$ & $2.69(1.52)$ & $2.49(1.13)$ & $2.69(1.37)$ & $3.30(0.95)$ \\
\hline & Week 52 (LOCF) & $1.27(0.85)$ & $1.58(1.03)$ & $1.24(0.87)$ & $1.09(0.55)$ & $1.68(1.12)$ \\
\hline & \% Change & $-49.7(25.7)^{* * *}$ & $-40.5(33.2)^{* * *}$ & $-48.9(26.5)^{* * *}$ & $-57.0(15.9)^{* * *}$ & $-51.0(22.4)^{* * *}$ \\
\hline \multirow{4}{*}{ LDL-C (mmol/L) } & $n$ & 189 & 21 & 123 & 34 & 8 \\
\hline & Baseline & $3.09(0.82)$ & $3.20(0.88)$ & $3.08(0.78)$ & $2.96(0.72)$ & $3.42(1.18)$ \\
\hline & Week 52 (LOCF) & $3.02(0.75)$ & $3.12(0.89)$ & $3.03(0.76)$ & $2.97(0.62)$ & $2.82(0.85)$ \\
\hline & \% Change & $2.2(30.4)$ & $2.7(33.7)$ & $2.1(29.2)$ & $5.1(30.0)$ & $-8.8(41.2)$ \\
\hline \multirow{4}{*}{ non HDL-C (mmol/L) } & $n$ & 189 & 21 & 123 & 34 & 8 \\
\hline & Baseline & $4.03(0.79)$ & $4.18(0.87)$ & $4.00(0.73)$ & $3.92(0.61)$ & $4.54(1.34)$ \\
\hline & Week 52 (LOCF) & $3.63(0.83)$ & $3.77(1.01)$ & $3.64(0.85)$ & $3.56(0.66)$ & $3.49(0.96)$ \\
\hline & $\%$ Change & $-8.7(18.8)^{* * *}$ & $-8.3(21.1)$ & $-8.4(17.4)^{* * *}$ & $-7.7(18.0)$ * & $-17.3(33.5)$ \\
\hline \multirow{4}{*}{ RemL-C (mmol/L) } & $n$ & 187 & 21 & 122 & 34 & 7 \\
\hline & Baseline & $0.48(0.26)$ & $0.54(0.30)$ & $0.46(0.26)$ & $0.48(0.26)$ & $0.59(0.15)$ \\
\hline & Week 52 (LOCF) & $0.18(0.13)$ & $0.21(0.15)$ & $0.18(0.14)$ & $0.17(0.09)$ & $0.24(0.17)$ \\
\hline & $\%$ Change & $-57.2(28.7)^{* * *}$ & $-59.9(21.1)^{* * *}$ & $-56.0(31.8)^{* * *}$ & $-59.3(22.9)^{* * *}$ & $-57.1(25.8)^{* *}$ \\
\hline \multirow{4}{*}{ apoA1 (mg/dL) } & $n$ & 187 & 21 & 122 & 34 & 7 \\
\hline & Baseline & $131.6(19.8)$ & $134.2(22.0)$ & $132.2(18.2)$ & $133.5(20.0)$ & $105.4(19.7)$ \\
\hline & Week 52 (LOCF) & $137.6(22.4)$ & $133.8(26.0)$ & $137.2(20.0)$ & $144.0(25.5)$ & $122.4(20.8)$ \\
\hline & $\%$ Change & $5.0(11.2) * * *$ & $0.0(13.9)$ & $4.1(10.5)^{* * *}$ & $8.0(10.0)^{* * *}$ & $16.7(10.6)^{* *}$ \\
\hline
\end{tabular}


Table 2. Cont.

\begin{tabular}{|c|c|c|c|c|c|c|}
\hline \multirow{2}{*}{\multicolumn{2}{|c|}{ Parameter }} & \multirow{2}{*}{ All Participants } & \multicolumn{4}{|c|}{ Baseline eGFR Category } \\
\hline & & & G1 & G2 & G3a-G3b & G4-G5 \\
\hline \multirow{4}{*}{ apoA2 (mg/dL) } & $n$ & 187 & 21 & 122 & 34 & 7 \\
\hline & Baseline & $30.3(4.5)$ & $31.4(5.1)$ & $30.8(4.2)$ & $29.5(4.7)$ & $24.2(3.3)$ \\
\hline & Week 52 (LOCF) & $38.2(6.9)$ & $37.9(5.9)$ & $38.7(6.8)$ & $37.8(6.8)$ & $32.8(7.1)$ \\
\hline & $\%$ Change & $27.0(19.5)^{* * *}$ & $22.2(18.4)^{* * *}$ & $26.3(17.6)^{* * *}$ & $29.4(22.7)^{* * *}$ & $35.3(21.7) * *$ \\
\hline \multirow{4}{*}{$\operatorname{apoB}(\mathrm{mg} / \mathrm{dL})$} & $n$ & 187 & 21 & 122 & 34 & 7 \\
\hline & Baseline & $93.6(16.8)$ & $95.6(17.8)$ & $93.4(16.5)$ & $91.9(13.8)$ & $97.4(23.5)$ \\
\hline & Week 52 (LOCF) & $88.1(18.6)$ & $93.0(23.1)$ & $88.0(18.5)$ & $87.9(15.4)$ & $76.1(21.6)$ \\
\hline & $\%$ Change & $-4.5(19.9)^{* *}$ & $-1.9(19.6)$ & $-4.5(19.6) *$ & $-3.0(17.7)$ & $-18.1(29.5)$ \\
\hline \multirow{4}{*}{$\operatorname{apoB} 48(\mu \mathrm{g} / \mathrm{mL})$} & $n$ & 188 & 21 & 123 & 34 & 7 \\
\hline & Baseline & $9.1(5.8)$ & $10.3(6.7)$ & $8.5(5.4)$ & $9.1(5.6)$ & $12.8(4.1)$ \\
\hline & Week 52 (LOCF) & $3.8(3.0)$ & $3.7(2.1)$ & $3.3(2.8)$ & $4.0(2.1)$ & $7.8(4.1)$ \\
\hline & $\%$ Change & $-53.0(30.9)^{* * *}$ & $-60.4(21.5)^{* * *}$ & $-54.4(33.0)^{* * *}$ & $-48.1(26.6)^{* * *}$ & $-34.9(34.4)$ * \\
\hline \multirow{4}{*}{ apoC3 (mg/dL) } & $n$ & 187 & 21 & 122 & 34 & 7 \\
\hline & Baseline & $15.1(4.5)$ & $15.9(5.0)$ & 14.7 (4.3) & $15.9(5.2)$ & $13.2(1.1)$ \\
\hline & Week 52 (LOCF) & $9.8(3.2)$ & $10.6(3.8)$ & $9.3(3.1)$ & $10.5(2.5)$ & $10.5(2.9)$ \\
\hline & $\%$ Change & $-32.4(19.6)^{* * *}$ & $-32.4(15.3)^{* * *}$ & $-34.2(20.1)^{* * *}$ & $-30.0(18.3)^{* * *}$ & $-20.2(22.6)$ \\
\hline \multirow{4}{*}{ hsCRP (mg/dL) } & n & 186 & 21 & 123 & 33 & 6 \\
\hline & Baseline & $0.11(0.20)$ & $0.18(0.43)$ & $0.09(0.11)$ & $0.13(0.19)$ & $0.23(0.39)$ \\
\hline & Week 52 (LOCF) & $0.13(0.26)$ & $0.23(0.58)$ & $0.12(0.19)$ & $0.13(0.15)$ & $0.06(0.03)$ \\
\hline & Change & $0.02(0.30)$ & $0.04(0.71)$ & $0.03(0.17)$ & $-0.00(0.22)$ & $-0.17(0.37)$ \\
\hline \multirow{4}{*}{$\mathrm{IL}-1 \beta(\mathrm{pg} / \mathrm{mL})$} & $n$ & 183 & 21 & 123 & 31 & 5 \\
\hline & Baseline & $0.16(0.22)$ & $0.21(0.28)$ & $0.15(0.20)$ & $0.17(0.28)$ & $0.13(0.10)$ \\
\hline & Week 52 (LOCF) & $0.09(0.08)$ & $0.08(0.08)$ & $0.08(0.08)$ & $0.11(0.10)$ & $0.13(0.12)$ \\
\hline & Change & $-0.07(0.24)^{* * *}$ & $-0.13(0.31)^{*}$ & $-0.06(0.22)^{* * *}$ & $-0.06(0.30)$ & $-0.00(0.17)$ \\
\hline
\end{tabular}

Data are presented as means (SD). Baseline eGFR categories are as follows: G1, eGFR $\geq 90 \mathrm{~mL} / \mathrm{min} / 1.73 \mathrm{~m}^{2}$; G2, eGFR $\geq 60 \mathrm{and}<90 \mathrm{~mL} / \mathrm{min} / 1.73 \mathrm{~m}{ }^{2}$; G3a-G3b, eGFR $\geq 30$ and $<60 \mathrm{~mL} / \mathrm{min} / 1.73 \mathrm{~m}^{2} ; \mathrm{G} 4-\mathrm{G} 5$, eGFR $<30 \mathrm{~mL} / \mathrm{min} / 1.73 \mathrm{~m}^{2}$. Three patients in hemodialysis were included in the all participants category but were excluded from the G4-G5 category. ${ }^{*} p<0.05,{ }^{* *} p<0.01,{ }^{* * *} p<0.001$ vs. baseline by Wilcoxon signed-rank tests for hsCRP and IL-1 $\beta$, and by one sample $t$-tests for all others. LOCF, last-observation-carried-forward; eGFR, estimated glomerular filtration rate; TG, triglyceride; HDL-C, high-density lipoprotein cholesterol; LDL-C, low-density lipoprotein cholesterol; RemL-C, remnant lipoprotein cholesterol; Apo, apolipoprotein; hsCRP, high-sensitivity C-reactive protein; IL-1 $\beta$, interleukin $1 \beta$; SD, standard deviation. 
Table 3. Changes from baseline in the level of cholesterol in CM, VLDL, LDL, and HDL subclasses measured by HPLC.

\begin{tabular}{|c|c|c|c|c|c|c|}
\hline \multicolumn{2}{|c|}{ Parameter } & \multirow{3}{*}{$\begin{array}{c}\text { All Participants } \\
188\end{array}$} & \multicolumn{4}{|c|}{ Baseline eGFR Category } \\
\hline & & & \multirow{2}{*}{$\begin{array}{c}\text { G1 } \\
21\end{array}$} & \multirow{2}{*}{$\begin{array}{l}\text { G2 } \\
123 \\
\end{array}$} & \multirow{2}{*}{$\begin{array}{c}\text { G3a-G3b } \\
34\end{array}$} & \multirow{2}{*}{$\begin{array}{c}\text { G4-G5 } \\
7\end{array}$} \\
\hline$(\mathrm{mmol} / \mathrm{L})$ & $n$ & & & & & \\
\hline \multirow{3}{*}{$\mathrm{CM}-\mathrm{C}$} & Baseline & $0.154(0.135)$ & $0.158(0.114)$ & $0.151(0.146)$ & $0.156(0.121)$ & $0.188(0.082)$ \\
\hline & Week 52 (LOCF) & $0.039(0.038)$ & $0.064(0.055)$ & $0.035(0.036)$ & $0.035(0.025)$ & $0.039(0.037)$ \\
\hline & $\%$ Change & $-64.3(35.5)^{* * *}$ & $-54.1(46.0) * * *$ & $-63.9(36.1)^{* * * *}$ & $-67.7(29.1)^{* * *}$ & $-79.4(14.0)^{* * *}$ \\
\hline \multirow{3}{*}{ VLDL-C } & Baseline & $1.196(0.335)$ & $1.260(0.390)$ & $1.164(0.300)$ & $1.177(0.319)$ & $1.559(0.474)$ \\
\hline & Week 52 (LOCF) & $0.866(0.264)$ & $0.932(0.323)$ & $0.834(0.234)$ & $0.892(0.293)$ & $1.076(0.372)$ \\
\hline & $\%$ Change & $-24.9(22.5)^{* * *}$ & $-24.6(20.0)^{* * *}$ & $-25.3(22.9)^{* * * *}$ & $-22.9(21.5)^{* * *}$ & $-28.7(25.5)^{*}$ \\
\hline \multirow{3}{*}{ Large LDL-C } & Baseline & $0.504(0.185)$ & $0.529(0.186)$ & $0.498(0.175)$ & $0.489(0.182)$ & $0.570(0.279)$ \\
\hline & Week 52 (LOCF) & $0.748(0.183)$ & $0.724(0.130)$ & $0.765(0.200)$ & $0.720(0.150)$ & $0.689(0.143)$ \\
\hline & $\%$ Change & $63.1(63.2)^{* * *}$ & $54.5(62.1)^{* * *}$ & $67.7(68.1)^{* * *}$ & $59.1(44.2)^{* * *}$ & $39.9(61.3)$ \\
\hline \multirow{3}{*}{ Medium LDL-C } & Baseline & $1.102(0.330)$ & $1.134(0.325)$ & $1.120(0.337)$ & $1.030(0.295)$ & $1.058(0.382)$ \\
\hline & Week 52 (LOCF) & $1.194(0.300)$ & $1.244(0.349)$ & $1.226(0.307)$ & $1.127(0.208)$ & $0.920(0.248)$ \\
\hline & \% Change & $17.3(49.9)^{* * *}$ & $15.5(37.9)$ & $18.7(53.7)^{* * *}$ & $18.9(45.1)^{*}$ & $-2.8(44.2)$ \\
\hline \multirow{3}{*}{ Small LDL-C } & Baseline & $0.682(0.190)$ & $0.711(0.236)$ & $0.690(0.187)$ & $0.650(0.160)$ & $0.643(0.223)$ \\
\hline & Week 52 (LOCF) & $0.517(0.165)$ & $0.576(0.223)$ & $0.522(0.158)$ & $0.501(0.139)$ & $0.381(0.154)$ \\
\hline & $\%$ Change & $-20.6(28.6)^{* * *}$ & $-18.0(21.3)^{* * *}$ & $-20.6(29.3)^{* * *}$ & $-18.9(28.2)^{* * *}$ & $-38.6(23.9)^{* *}$ \\
\hline \multirow{3}{*}{ Very small LDL-C } & Baseline & $0.254(0.080)$ & $0.262(0.064)$ & $0.250(0.080)$ & $0.264(0.084)$ & $0.258(0.113)$ \\
\hline & Week 52 (LOCF) & $0.210(0.061)$ & $0.233(0.080)$ & $0.211(0.056)$ & $0.207(0.063)$ & $0.158(0.056)$ \\
\hline & \% Change & $-11.6(30.2)^{* * *}$ & $-10.3(23.2)$ & $-9.7(30.1)^{* * *}$ & $-15.8(29.0)^{* *}$ & $-33.4(21.4)^{* *}$ \\
\hline \multirow{3}{*}{ Very large HDL-C } & Baseline & $0.046(0.015)$ & $0.047(0.016)$ & $0.046(0.013)$ & $0.048(0.016)$ & $0.038(0.015)$ \\
\hline & Week 52 (LOCF) & $0.048(0.016)$ & $0.047(0.018)$ & $0.048(0.016)$ & $0.051(0.017)$ & $0.044(0.020)$ \\
\hline & \% Change & $4.4(21.5)^{* *}$ & $0.8(18.3)$ & $4.1(21.0)^{*}$ & $5.9(20.4)$ & $17.1(36.1)$ \\
\hline \multirow{3}{*}{ Large HDL-C } & Baseline & $0.130(0.083)$ & $0.123(0.103)$ & $0.130(0.080)$ & $0.139(0.082)$ & $0.096(0.085)$ \\
\hline & Week 52 (LOCF) & $0.115(0.088)$ & $0.107(0.100)$ & $0.115(0.088)$ & $0.124(0.082)$ & $0.096(0.090)$ \\
\hline & $\%$ Change & $-9.2(45.1)^{* *}$ & $-14.1(43.2)$ & $-10.9(44.7) * *$ & $-4.9(42.6)$ & $8.2(53.4)$ \\
\hline \multirow{3}{*}{ Medium HDL-C } & Baseline & $0.374(0.114)$ & $0.389(0.131)$ & $0.382(0.105)$ & $0.372(0.118)$ & $0.216(0.072)$ \\
\hline & Week 52 (LOCF) & $0.443(0.155)$ & $0.441(0.187)$ & $0.448(0.146)$ & $0.447(0.170)$ & $0.336(0.141)$ \\
\hline & \% Change & $19.3(24.4)^{* * *}$ & $11.4(21.3)^{*}$ & $17.7(23.9)^{* * *}$ & $20.6(22.3)^{* * *}$ & $53.2(25.3)^{* * *}$ \\
\hline \multirow{3}{*}{ Small HDL-C } & Baseline & $0.388(0.076)$ & $0.414(0.092)$ & $0.393(0.070)$ & $0.382(0.072)$ & $0.281(0.057)$ \\
\hline & Week 52 (LOCF) & $0.473(0.087)$ & $0.482(0.097)$ & $0.477(0.079)$ & $0.467(0.082)$ & $0.395(0.106)$ \\
\hline & $\%$ Change & $24.2(23.3) * * *$ & $18.1(16.8)^{* * *}$ & $23.6(23.3)^{* * *}$ & $24.6(22.4)^{* * * *}$ & $39.1(20.9)^{* *}$ \\
\hline \multirow{3}{*}{ Very small HDL-C } & Baseline & $0.148(0.032)$ & $0.158(0.032)$ & $0.146(0.034)$ & $0.154(0.023)$ & $0.128(0.035)$ \\
\hline & Week 52 (LOCF) & $0.181(0.033)$ & $0.186(0.035)$ & $0.181(0.031)$ & $0.180(0.024)$ & $0.155(0.042)$ \\
\hline & $\%$ Change & $27.9(36.6)^{* * *}$ & $20.6(25.0) * *$ & $30.9(39.7)^{* * *}$ & $19.7(22.8)^{* * *}$ & $23.5(25.9)$ \\
\hline
\end{tabular}

Data are presented as means (SD). Lipoprotein fractions were measured by HPLC at baseline and at week 12 and week 40. Baseline eGFR categories are as follows: G1, eGFR $\geq 90 \mathrm{~mL} / \mathrm{min} / 1.73 \mathrm{~m}^{2}$; G2, eGFR $\geq 60$ and $<90 \mathrm{~mL} / \mathrm{min} / 1.73 \mathrm{~m}^{2}$; G3a-G3b, eGFR $\geq 30$ and $<60 \mathrm{~mL} / \mathrm{min} / 1.73 \mathrm{~m}{ }^{2}$; G4-G5, eGFR $<30 \mathrm{~mL} / \mathrm{min} / 1.73 \mathrm{~m}{ }^{2}$. Three patients in hemodialysis were included in the all participants category but were excluded from the G4-G5 category. $*<<0.05, * *<0.01, * * *<0.001$ vs. baseline by one sample $t$-test. eGFR, estimated glomerular filtration rate; CM-C, chylomicron cholesterol; VLDL-C, very-low-density lipoprotein cholesterol; LDL-C, low-density lipoprotein cholesterol; HDL-C, high-density lipoprotein cholesterol; HPLC, high-performance liquid chromatography; LOCF, last-observation-carried-forward; SD, standard deviation 


\subsection{Safety}

The incidence of total AEs and adverse drug reactions (ADRs) over 52 weeks was $82.0 \%$ and $31.7 \%$, respectively (Table 4). AEs with an incidence $\geq 5 \%$ included nasopharyngitis $(28.0 \%$ ) and cholelithiasis (5.8\%). The incidence of AEs during weeks 0-12, 12-24, 24-36, 36-48, and 48-52 was $40.2 \%$ (76/189), 46.5\% (86/185), 39.2\% (71/181), 34.7\% (60/173), and $12.9 \%(22 / 171)$ of the patients, respectively, showing no increase over time. The incidence of total AEs/ADRs was similar across the baseline eGFR subgroups (Table 4).

Table 4. Summary of AEs and ADRs.

\begin{tabular}{|c|c|c|c|c|c|c|}
\hline \multirow{2}{*}{ Parameter } & \multirow{2}{*}{$\begin{array}{c}\text { All } \\
\text { Participants }\end{array}$} & \multicolumn{4}{|c|}{ Baseline eGFR Category } & \multirow{2}{*}{ Hemodialysis } \\
\hline & & G1 & G2 & G3a-G3b & G4-G5 & \\
\hline$n$ & 189 & 21 & 123 & 34 & 8 & 3 \\
\hline \multicolumn{7}{|l|}{$\mathrm{AE}$} \\
\hline Total & $155(82.0)$ & $17(81.0)$ & $97(78.9)$ & $31(91.2)$ & $7(87.5)$ & $3(100)$ \\
\hline Serious & $16(8.5)$ & $2(9.5)$ & $4(3.3)$ & $5(14.7)$ & $2(25.0)$ & $3(100)$ \\
\hline Leading to withdrawal & $11(5.8)$ & $2(9.5)$ & $3(2.4)$ & $3(8.8)$ & $3(37.5)$ & 0 \\
\hline \multicolumn{7}{|l|}{ ADR } \\
\hline Total & $60(31.7)$ & $9(42.9)$ & $34(27.6)$ & $15(44.1)$ & $2(25.0)$ & 0 \\
\hline Serious & $1(0.5)$ & 0 & $1(0.8)$ & 0 & 0 & 0 \\
\hline Leading to withdrawal & $7(3.7)$ & $2(9.5)$ & $2(1.6)$ & $1(2.9)$ & $2(25.0)$ & 0 \\
\hline $\mathrm{CK}>\mathrm{ULN} \times 2.5$ & $11(5.8)$ & $1(4.8)$ & $8(6.5)$ & $1(2.9)$ & $1(12.5)$ & 0 \\
\hline $\mathrm{CK}>\mathrm{ULN} \times 5$ & $1(0.5)$ & 0 & $1(0.8)$ & 0 & 0 & 0 \\
\hline $\mathrm{sCr}>$ Baseline $\times 2.0$ & 0 & 0 & 0 & 0 & 0 & 0 \\
\hline
\end{tabular}

Data are presented as the number of patients (\%). Baseline eGFR categories are as follows: G1, eGFR $\geq 90 \mathrm{~mL} / \mathrm{min} / 1.73 \mathrm{~m}^{2} ; \mathrm{G} 2$, eGFR $\geq 60$ and $<90 \mathrm{~mL} / \mathrm{min} / 1.73 \mathrm{~m}^{2} ; \mathrm{G} 3 \mathrm{a}-\mathrm{G} 3 \mathrm{~b}, \mathrm{eGFR} \geq 30$ and $<60 \mathrm{~mL} / \mathrm{min} / 1.73 \mathrm{~m}^{2}$; G4-G5, eGFR $<30 \mathrm{~mL} / \mathrm{min} / 1.73 \mathrm{~m}^{2}$. eGFR, estimated glomerular filtration rate; AE, adverse event; ADR, adverse drug reaction; $\mathrm{CK}$, creatine kinase; ULN, upper limit of normal; $\mathrm{sCr}$, serum creatinine.

Serious adverse events (SAEs) occurred in 8.5\% (16/189) of the patients (Tables 4 and 5). Death due to acute myocardial infarction occurred in one patient in the G2 subgroup. A causal relationship between SAEs and pemafibrate treatment was ruled out in all cases except for cerebral infarction in one patient in the G2 subgroup. All those who underwent hemodialysis experienced SAEs, including malaise in one patient and shunt occlusion/stenosis in two others, of which one also experienced cataracts and upper respiratory tract inflammation. AEs leading to discontinuation occurred in $5.8 \%(11 / 189)$ of the patients (Tables 4 and 5) and the incidence rate was highest in the G4-G5 subset of this group [37.5\% (3/8)]. Relevant events included aortic aneurysm/dissection and carotid artery dissection, chronic kidney disease, and drug eruption in three different patients. 
Table 5. Preferred terms of AEs (underlined events are ADRs).

\begin{tabular}{|c|c|c|c|c|c|c|c|c|c|c|c|}
\hline \multirow{2}{*}{ AEs/ADRs } & \multirow{2}{*}{ All } & \multicolumn{8}{|c|}{ Baseline eGFR Category } & \multirow{2}{*}{\multicolumn{2}{|c|}{ Hemodialysis }} \\
\hline & & \multicolumn{2}{|r|}{ G1 } & \multicolumn{2}{|r|}{ G2 } & \multicolumn{2}{|c|}{ G3a-G3b } & \multicolumn{2}{|r|}{ G4-G5 } & & \\
\hline$n$ & 189 & \multicolumn{2}{|r|}{21} & \multicolumn{2}{|r|}{123} & \multicolumn{2}{|r|}{34} & \multicolumn{2}{|r|}{8} & \multicolumn{2}{|r|}{3} \\
\hline \multirow[t]{6}{*}{ Serious } & \multirow[t]{6}{*}{16} & \multicolumn{2}{|r|}{2} & \multicolumn{2}{|r|}{4} & \multicolumn{2}{|r|}{5} & \multicolumn{2}{|r|}{2} & \multicolumn{2}{|r|}{3} \\
\hline & & G1-1 & $\begin{array}{l}\text { Diabetes } \\
\text { mellitus }\end{array}$ & G2-1 & $\begin{array}{l}\text { Inguinal hernia } \\
\text { Cryptorchism }\end{array}$ & G3-1 & Aortic aneurysm & G45-1 & $\begin{array}{c}\text { Gastric adenoma } \\
\text { Adenocarcinoma gastric }\end{array}$ & $\mathrm{H}-1$ & $\begin{array}{l}\text { Shunt occlusion } \\
\text { Shunt stenosis }\end{array}$ \\
\hline & & \multirow[t]{4}{*}{ G1-2 } & \multirow[t]{4}{*}{$\begin{array}{l}\text { Myocardial } \\
\text { ischemia }\end{array}$} & G2-2 & Sepsis & G3-2 & $\begin{array}{l}\text { Adenocarcinoma } \\
\text { gastric }\end{array}$ & \multirow{4}{*}{ G45-2 } & \multirow{4}{*}{$\begin{array}{l}\text { Spinal compression } \\
\text { fracture } \\
\text { Aortic aneurysm } \\
\text { Aortic dissection } \\
\text { Carotid artery dissection }\end{array}$} & $\mathrm{H}-2$ & Malaise \\
\hline & & & & G2-3 & Cerebral infarction & G3-3 & Pneumonia & & & & \\
\hline & & & & G2-4 & $\begin{array}{l}\text { Acute myocardial } \\
\text { infarction }\end{array}$ & G3-4 & Pneumonia & & & H-3 & $\begin{array}{l}\text { Shunt stenosis } \\
\text { Cataract } \\
\text { Unper respiratory }\end{array}$ \\
\hline & & & & & & G3-5 & $\begin{array}{l}\text { Myocardial } \\
\text { ischemia }\end{array}$ & & & & tract inflammation \\
\hline $\begin{array}{l}\text { Leading to } \\
\text { withdrawal }\end{array}$ & 11 & & 2 & & 3 & & 3 & & 3 & & 0 \\
\hline & & G1-3 & $\underline{\text { Cholelithiasis }}$ & G2-5 & $\begin{array}{l}\text { AST increased } \\
\text { ALT increased } \\
\end{array}$ & G3-2 & $\begin{array}{l}\text { Adenocarcinoma } \\
\text { gastric }\end{array}$ & G45-2 & $\begin{array}{l}\text { Aortic aneurysm } \\
\text { Aortic dissection }\end{array}$ & & \\
\hline & & G1-4 & Diabetes mellitus & G2-6 & Cholelithiasis & G3-3 & Pneumonia & & Carotid artery dissection & & \\
\hline & & & & & Acute myocardial & & LDL increased & G45-3 & Chronic kidney disease & & \\
\hline & & & & G2-4 & infarction & G3-6 & Cholelithiasis & G45-4 & Drug eruption & & \\
\hline
\end{tabular}


No rhabdomyolysis was observed. Myalgia was reported in 2.6\% (5/189) of the patients. Four of these patients were in the G2 subgroup and one was in the G3a-G3b subgroup, where three patients and one patient received concomitant statin therapy in each group, respectively. There were no significant changes in creatine kinase (CK) levels over time in any eGFR subgroup (Figure 5 and Table 6). Increases in CK to $>2.5 \times$ ULN (upper limit of normal) occurred in $5.8 \%(11 / 189)$ of the patients, and this was evenly distributed across the eGFR subgroups (Table 4), where two patients with baseline eGFR $<60 \mathrm{~mL} / \mathrm{min} / 1.73 \mathrm{~m}^{2}$ and four out of nine patients with baseline eGFR $\geq 60 \mathrm{~mL} / \mathrm{min} / 1.73 \mathrm{~m}^{2}$ were not taking statins. Among them, one patient who was not on statin therapy in the G2 subgroup increased CK to $>5 \times$ ULN. Specifically, the CK level of this patient increased from $215 \mathrm{U} / \mathrm{L}$ at baseline to $1452 \mathrm{U} / \mathrm{L}$ at week 4 and then decreased to $156 \mathrm{U} / \mathrm{L}$ at week 8 without discontinuing pemafibrate treatment. No additional significant changes in CK level were noted until week 52 . There were no major changes in eGFR over time in any eGFR subgroup except for a decrease from $99.6 \mathrm{~mL} / \mathrm{min} / 1.73 \mathrm{~m}^{2}$ at baseline to $91.8 \mathrm{~mL} / \mathrm{min} / 1.73 \mathrm{~m}^{2}$ after 8 weeks in the G1 group (Figure 5). No patients presented increases in serum creatinine $>2 \times$ the baseline value (Table 4). $\gamma$-GT and alkaline phosphatase (ALP) levels significantly decreased in the G1, G2, and G3a-G3b groups (Table 6).
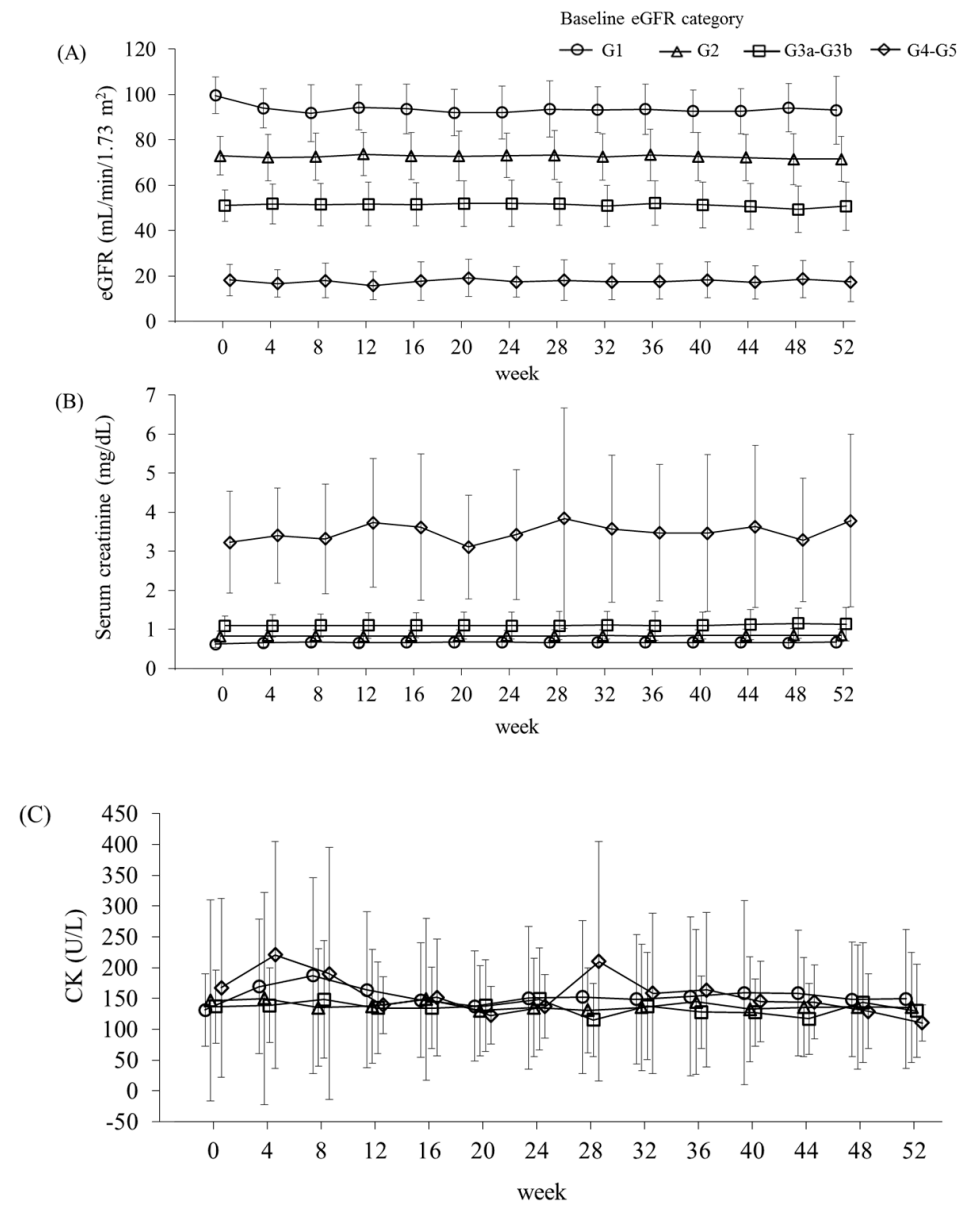

$\begin{array}{ccccccccccccccc}\text { No. of patients } & & & & & & & & & & & & & & \\ \text { G1 } & 21 & 21 & 21 & 21 & 21 & 21 & 21 & 21 & 21 & 21 & 20 & 19 & 19 & 19 \\ \text { G2 } & 123 & 123 & 122 & 121 & 121 & 121 & 121 & 121 & 119 & 117 & 116 & 116 & 116 & 116 \\ \text { G3a-G3b } & 34 & 34 & 33 & 32 & 33 & 32 & 31 & 30 & 29 & 29 & 29 & 29 & 27 & 28\end{array}$

$\begin{array}{ccccccccccccccc}\text { G3a-G3b } & 34 & 34 & 33 & 32 & 33 & 32 & 31 & 30 & 29 & 29 & 29 & 29 & 27 & 28 \\ \text { G4-G5 } & 8 & 8 & 8 & 6 & 7 & 5 & 5 & 5 & 5 & 5 & 5 & 5 & 5 & 5\end{array}$

Figure 5. Time course of the levels of eGFR (A), serum creatinine (B), and CK (C) by baseline eGFR category. Baseline eGFR categories are as follows: G1, eGFR $\geq 90 \mathrm{~mL} / \mathrm{min} / 1.73 \mathrm{~m}^{2}$; G2, eGFR $\geq 60$ and $<90 \mathrm{~mL} / \mathrm{min} / 1.73 \mathrm{~m}^{2}$; G3a-G3b, eGFR $\geq 30$ and $<60 \mathrm{~mL} / \mathrm{min} / 1.73 \mathrm{~m}^{2}$; and G4-G5, eGFR $<30 \mathrm{~mL} / \mathrm{min} / 1.73 \mathrm{~m}^{2}$. Data are presented as means \pm SD. eGFR, estimated glomerular filtration rate; $\mathrm{CK}$, creatine kinase; $\mathrm{SD}$, standard deviation. 
Table 6. Changes from baseline in the levels of safety parameters.

\begin{tabular}{|c|c|c|c|c|c|c|}
\hline \multirow{2}{*}{ Parameter } & & \multirow{2}{*}{ All Participants } & \multicolumn{4}{|c|}{ Baseline eGFR Category } \\
\hline & & & G1 & G2 & G3a-G3b & G4-G5 \\
\hline \multirow{3}{*}{ AST (U/L) } & $n$ & 166 & 18 & 113 & 27 & 5 \\
\hline & Week 52 & $25.8(12.0)$ & $28.7(16.2)$ & $26.1(12.4)$ & $24.7(6.3)$ & $21.6(5.9)$ \\
\hline & Change & $-1.5(11.3) *$ & $2.5(14.9)$ & $-1.7(10.5) *$ & $-4.7(12.6) *$ & $3.6(3.0)$ \\
\hline \multirow{2}{*}{$\operatorname{ALT}(\mathrm{U} / \mathrm{L})$} & $n$ & 171 & 19 & 116 & 28 & 5 \\
\hline & Change & $-8.0(13.7)^{* * *}$ & $-3.3(13.1)$ & $-9.2(14.6)^{* * *}$ & $-8.3(10.8)^{* * *}$ & $1.6(6.2)$ \\
\hline \multirow{4}{*}{$\gamma-\mathrm{GT}(\mathrm{U} / \mathrm{L})$} & $n$ & 171 & 19 & 116 & 28 & 5 \\
\hline & Baseline & $55.0(52.3)$ & $73.6(96.6)$ & $55.2(41.3)$ & $50.8(56.3)$ & $22.2(10.4)$ \\
\hline & Week 52 & $32.2(35.0)$ & $48.6(66.2)$ & $32.3(31.3)$ & $25.0(16.3)$ & $18.0(14.2)$ \\
\hline & Change & $-22.8(31.7)^{* * *}$ & $-24.9(37.8)^{* *}$ & $-22.9(28.4)^{* * *}$ & $-25.8(42.7)^{* * *}$ & $-4.2(8.2)$ \\
\hline \multirow{4}{*}{ Serum creatinine $(\mathrm{mg} / \mathrm{dL})$} & $n$ & 171 & 19 & 116 & 28 & 5 \\
\hline & Baseline & $1.02(0.98)$ & $0.62(0.10)$ & $0.83(0.13)$ & $1.08(0.27)$ & $2.98(1.19)$ \\
\hline & Week 52 & 1.09 (1.23) & $0.67(0.14)$ & $0.85(0.16)$ & $1.13(0.43)$ & $3.78(2.21)$ \\
\hline & Change & $0.07(0.34)^{* * *}$ & $0.06(0.09)^{* *}$ & $0.02(0.07)^{* *}$ & $0.06(0.21)$ & $0.80(1.26)$ \\
\hline \multirow{4}{*}{$\mathrm{eGFR}\left(\mathrm{mL} / \mathrm{min} / 1.73 \mathrm{~m}^{2}\right)$} & $n$ & 171 & 19 & 116 & 28 & 5 \\
\hline & Baseline & $69.8(19.5)$ & $100.3(8.1)$ & $73.1(8.5)$ & $51.2(7.2)$ & $19.2(6.7)$ \\
\hline & Week 52 & $67.8(19.6)$ & $93.0(14.8)$ & $71.5(10.0)$ & $50.7(10.8)$ & $17.4(8.8)$ \\
\hline & Change & $-2.0(7.2)^{* * *}$ & $-7.2(11.9) *$ & $-1.5(6.1)^{*}$ & $-0.5(6.9)$ & $-1.8(4.9)$ \\
\hline \multirow{4}{*}{ CK $(\mathrm{U} / \mathrm{L})$} & $n$ & 171 & 19 & 116 & 28 & 5 \\
\hline & Baseline & $142.4(142.3)$ & $132.1(61.5)$ & $149.2(168.0)$ & $132.6(60.5)$ & $113.6(34.4)$ \\
\hline & Week 52 & $134.3(88.4)$ & $149.3(113.0)$ & $135.5(89.6)$ & $129.4(75.8)$ & $110.2(29.5)$ \\
\hline & Change & $-8.1(139.9)$ & $17.2(74.0)$ & $-13.7(163.9)$ & $-3.2(67.3)$ & $-3.4(15.1)$ \\
\hline
\end{tabular}

Data are presented as means (SD). Baseline eGFR categories are as follows: G1, eGFR $\geq 90 \mathrm{~mL} / \mathrm{min} / 1.73 \mathrm{~m}^{2}$; G2, eGFR $\geq 60$ and $<90 \mathrm{~mL} / \mathrm{min} / 1.73 \mathrm{~m}{ }^{2}$; G3a-G3b, eGFR $\geq 30$ and $<60 \mathrm{~mL} / \mathrm{min} / 1.73 \mathrm{~m}^{2} ; \mathrm{G} 4-\mathrm{G} 5$, eGFR $<30 \mathrm{~mL} / \mathrm{min} / 1.73 \mathrm{~m}^{2}$. Three patients in hemodialysis were included in all participants but were excluded from the G4-G5 category. ${ }^{*} p<0.05$, ${ }^{* *} p<0.01,{ }^{* * *} p<0.001$ vs. baseline by Wilcoxon signed-rank test. eGFR, estimated glomerular filtration rate; AST, aspartate aminotransferase; ALT, alanine aminotransferase; $\gamma$-GT, $\gamma$-glutamyltransferase; ALP, alkaline phosphatase; CK, creatine kinase; SD, standard deviation. 


\subsection{Plasma Concentrations}

Plasma concentrations of pemafibrate were obtained from 114 patients among the 189 participants; one had only the trough value. Blood sampling after pemafibrate administration was conducted at week 4 in 23 patients, week 8 in 38 patients, week 12 in 21 patients, week 16 in 14 patients, week 20 in 12 patients, and week 24 in five patients, with the largest number of blood samples collected during week 8 . At every trough sampling time and at $0.5-<1.5 \mathrm{~h}, 1.5-3 \mathrm{~h}$, and $4-6 \mathrm{~h}$ after pemafibrate administration, the pemafibrate plasma concentrations were comparable across the subgroups stratified by renal function (Figure 6).
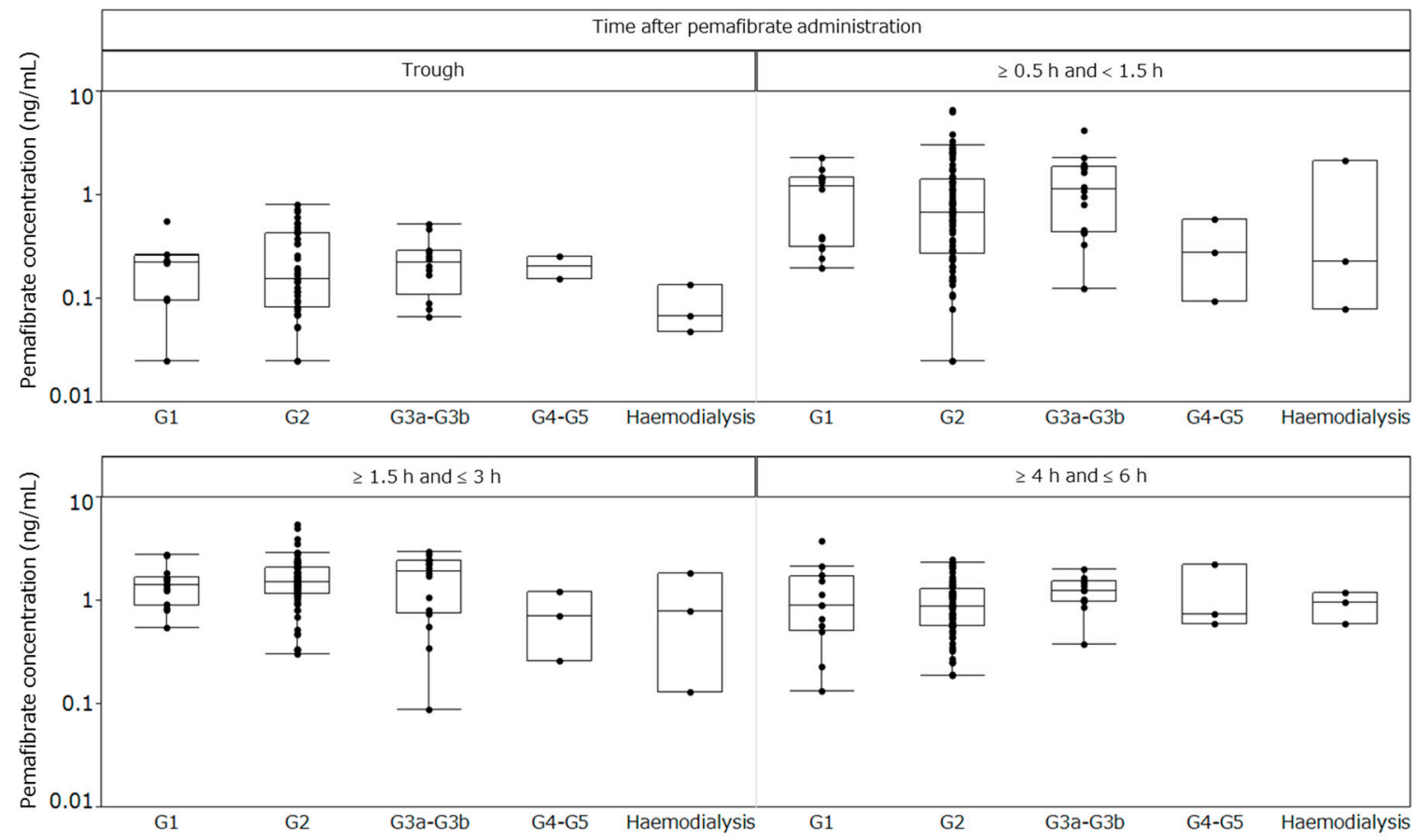

Figure 6. Plasma concentrations of pemafibrate after repeated-dose administration. Baseline eGFR categories are as follows: G1, eGFR $\geq 90 \mathrm{~mL} / \mathrm{min} / 1.73 \mathrm{~m}^{2} ; \mathrm{G} 2, \mathrm{eGFR} \geq 60$ and $<90 \mathrm{~mL} / \mathrm{min} / 1.73 \mathrm{~m}^{2}$; G3a-G3b, eGFR $\geq 30$ and $<60 \mathrm{~mL} / \mathrm{min} / 1.73 \mathrm{~m}^{2}$; G4-G5, eGFR $<30 \mathrm{~mL} / \mathrm{min} / 1.73 \mathrm{~m}^{2}$. eGFR, estimated glomerular filtration rate.

\section{Discussion}

In the present phase III trial, a broad range of patients-including those with impaired renal function (CKD stages G3a, G3b, G4, and G5, and receiving hemodialysis)—was recruited, and the duration of administration (52 weeks) was longer than that in previous pemafibrate trials. Compared to the baseline, pemafibrate reduced fasting serum TG levels by $\sim 50 \%$. There were no significant differences in TG reduction across the prespecified subgroups stratified by baseline patient characteristics. Pemafibrate uptitration from $0.2 \mathrm{mg} /$ day to $0.4 \mathrm{mg} /$ day further reduced TG levels. There were no time-dependent increases in the incidence of AEs/ADRs during long-term pemafibrate administration. Furthermore, the overall efficacy and safety of pemafibrate were similar across all subgroups stratified by baseline eGFR.

The efficacy of pemafibrate to ameliorate an abnormal lipid profile has been demonstrated in previous clinical studies of up to 24 weeks long and was also observed in the present 52-week trial [22-26]. Patients with impaired renal function often have atherogenic dyslipidemia, including high TG and low HDL-C levels, which occurs as a result of impaired TG-rich lipoprotein catabolism associated with decreased lipoprotein lipase (LPL) activity or impaired HDL maturation caused by reduced lecithin-cholesterol acyltransferase (LCAT) activity [7,32-35]. In addition, apoA1 and apoA2 levels are decreased in these patients [36]. In the present study, although baseline TG levels were not 
correlated with baseline eGFR levels, baseline TG-rich lipoprotein cholesterol levels (as indicated by CM-C and VLDL-C) were highest in the G4-G5 subgroup. Pemafibrate achieved the greatest reductions in TG-rich lipoprotein levels in the subgroups with low baseline eGFR. In contrast, pemafibrate caused the largest increase in HDL-C, apoA1, and apoA2 levels in the lowest eGFR subgroup. Pemafibrate treatment also resulted in the greatest decreases in small LDL-C and very small LDL-C particles in the subgroup with the lowest baseline eGFR. Therefore, pemafibrate may have substantial influences on the atherogenic lipoprotein profiles frequently observed in patients with impaired renal function. Clarification of the underlying mechanism of these findings will require further investigation.

The present study had no control group. Therefore, it could not be determined whether the incidences of AEs and ADRs in patients receiving pemafibrate were higher or lower than those receiving other treatments or placebo. According to an earlier report, hypertriglyceridemic patients with normal renal function receiving pemafibrate treatment had lower incidences of AEs and ADRs than those receiving fenofibrate [24,26]. The AE and ADR incidences and profiles in patients administered pemafibrate alone or in combination with a statin were comparable to those observed in the placebo group $[22,23,25,26]$.

The major AEs of concern associated with fibrate administration are rhabdomyolysis/myopathy and renal function decline [15]. The risk of rhabdomyolysis/myopathy may increase when fibrates are administered in combination with statins [20]. However, no cases of rhabdomyolysis were observed in the present study. CK elevation $>5 \times$ ULN was observed in only one patient in the G2 subgroup. The incidences of myalgia and CK elevation $>2.5 \times$ ULN were not correlated with baseline eGFR. Moreover, none of these AEs led to treatment discontinuation. CK levels did not significantly increase in patients with reduced renal function. However, the number of patients with baseline eGFR $<60 \mathrm{~mL} / \mathrm{min} / 1.73 \mathrm{~m}^{2}$ in this study was limited; thus, larger-scale trials are required to confirm the safety of pemafibrate in patients with CKD.

Here, pemafibrate treatment was shown to significantly increase serum creatinine levels in the G1 and G2 subgroups with mean changes of 0.06 and $0.02 \mathrm{mg} / \mathrm{dL}$, respectively. These increases are comparable to those observed in previous studies but are lower than those reported for fenofibrate [22-26]. Furthermore, there were no significant changes in eGFR in the G3a-G3b and G4-G5 subgroups.

In this study, we treated three patients undergoing hemodialysis for 52 weeks. In hemodialysis patients, LDL-C levels are not high, but lipid abnormalities such as high TG, high small LDL particles, and low HDL-C may occur [7]. Conventional fibrates are contraindicated in patients with severe renal dysfunction [37]. However, several reports have evaluated the efficacy and safety of fibrates in patients receiving dialysis [38-40]. In the present study, the TG reductions in the three patients receiving hemodialysis were comparable to those in the total population, and although AEs occurred in all three patients on hemodialysis, none of these AEs were deemed to be related to pemafibrate treatment. However, pemafibrate efficacy and safety data are limited for hemodialysis patients; therefore, further studies are necessary to validate the usefulness of pemafibrate administration in hemodialysis patients.

The patient who died of an acute myocardial infarction was a smoker and had undergone percutaneous coronary intervention before participating in the present study. Therefore, the investigator concluded that this particular $\mathrm{AE}$ was not associated with pemafibrate treatment. A causal relationship between pemafibrate and one cerebral infarction event could not be excluded by the investigator. This patient had hypertension, fatty liver, and type 2 diabetes mellitus, and smoked $\sim 20$ cigarettes/day. Consequently, this patient was most likely already at increased risk of cerebral infarction.

After oral administration of ${ }^{14} \mathrm{C}$-pemafibrate, pemafibrate was mainly excreted into the bile with very little urinary excretion of unchanged pemafibrate $(<0.5 \%)[27,30]$. Moreover, a pharmacokinetic study of a single oral administration of pemafibrate in patients with normal and impaired renal function showed no increase in systemic exposure in a renal function-dependent manner [27,31]. Consistent with these findings, the present study showed no increase in the plasma concentration 
of pemafibrate in patients with severely impaired renal function or those on dialysis compared with that in patients with normal renal function, even in the steady state after repeated administration. Although patients with impaired renal function frequently have comorbidities such as elevated TG and low HDL-C levels, precautions are required for the use of conventional fibrate drugs because they are primarily excreted via the kidneys, and their plasma concentrations increase in patients with impaired renal function [41-46]. Therefore, pemafibrate may offer a new therapeutic option for dyslipidemic patients with impaired renal function.

The limitations of the present study are as follows: (1) this study had no control group, and the presence of impaired renal function by itself may have been associated with a higher occurrence of AEs. Therefore, a randomized, placebo-controlled trial is necessary to assess pemafibrate safety in patients with impaired renal function; (2) the number of patients in the G4-G5 subgroup was limited. A larger-scale study with enriched enrollment of such patients is needed to confirm pemafibrate efficacy and safety in this population; (3) the study participants were all Japanese. For this reason, it is unknown whether similar results would be found in other ethnic populations. In the U.S., a pemafibrate trial is currently underway involving patients with extremely high TG levels and mildly to moderately impaired renal function (NCT03011450).

\section{Subjects and Methods}

This was a multicenter, single-arm, open-label, phase III trial. It was conducted at 32 sites in Japan between May 2014 and November 2015. Written informed consent was obtained from the participants before trial initiation. The protocol was approved by the institutional review board. This trial was conducted in accordance with the principles of the Declaration of Helsinki and the "Ministerial Ordinance on Good Clinical Practice" (GCP Ordinance) and was registered with the Japan Pharmaceutical Information Center (JAPIC) (JapicCTI-142496, 2 April 2014).

The inclusion criteria were as follows: (1) patients with dyslipidemia aged $\geq 20$ years at the time of informed consent; (2) men and postmenopausal women; (3) fasting serum TG $\geq 1.70 \mathrm{mmol} / \mathrm{L}$ $(150 \mathrm{mg} / \mathrm{dL})$ at two consecutive measurements during screening; and (4) patients who followed dietary and physical exercise guidance for $\geq 12$ weeks before enrollment.

The major exclusion criteria were as follows: (1) patients with fasting serum TG $\geq 5.65 \mathrm{mmol} / \mathrm{L}$ (500 mg/dL) during screening; (2) patients with poorly controlled diabetes mellitus (hemoglobin A1c $[\mathrm{HbA} 1 \mathrm{c}] \geq 10.5 \%$ ); (3) patients with concurrent poorly controlled thyroid disease; (4) men with serum creatinine $\geq 2.5 \mathrm{mg} / \mathrm{dL}$ and women with serum creatinine $\geq 2.0 \mathrm{mg} / \mathrm{dL}$ during screening who were already on statin therapy; (5) patients with eGFR $<45 \mathrm{~mL} / \mathrm{min} / 1.73 \mathrm{~m}^{2}$ during screening who were already on statin therapy; (6) patients with CK $>5 \times \mathrm{ULN}$ ( $270 \mathrm{IU} / \mathrm{L}$ for men, $150 \mathrm{IU} / \mathrm{L}$ for women) during screening who were already on statin therapy; (7) patients with serious liver disease (cirrhosis Child-Pugh Class B or higher); (8) patients with gallstones or serious biliary tract disease; (9) patients who had suffered an acute myocardial infarction or stroke within three months before informed consent; and (10) patients with New York Heart Association class III or IV heart failure. During the trial period, the following drugs were prohibited from concomitant use: fibrates, p-glycoprotein inhibitors, breast cancer-resistance protein inhibitors, and organic anion transporting polypeptide 1B1 or 1B3 inhibitors. Initiation, discontinuation, and modification of the dosage regimens of corticosteroids, protease inhibitors, anabolic steroid hormones, progestogens, lipid lowering agents, or hypoglycemic agents other than the abovementioned drugs prohibited for concomitant use were essentially prohibited. However, the addition or dosage increase of lipid lowering agents and hypoglycemic agents was permitted if deemed necessary. The addition of lipid lowering agents and thiazolidinediones was prohibited for the first 12 weeks of the study so that their effects on drug efficacy could be assessed at week 12.

During the screening period (8 weeks prior to treatment initiation), tests were performed twice to determine patient eligibility. Thereafter, eligible patients orally received pemafibrate $0.2 \mathrm{mg} /$ day (twice daily) for 52 weeks. From week 12 of the treatment period onwards, the investigators were 
instructed that the dose could be increased from $0.2 \mathrm{mg} /$ day to $0.4 \mathrm{mg}$ /day (twice daily) if there was an inadequate response to the initial dose based on fasting serum TG levels $\geq 1.70 \mathrm{mmol} / \mathrm{L}(150 \mathrm{mg} / \mathrm{dL})$.

Fasting blood and urine samples were collected at each visit. Blood and urine sample in patients with hemodialysis were collected just before dialysis. LDL-C levels were measured using the direct method. Lipoprotein fractions were measured by HPLC [47] at baseline and weeks 12 and 40. All central measurements were made by LSI Medience Corp. (Chiyoda-ku, Tokyo, Japan) except for the lipoprotein fraction, which was determined by Skylight Biotech Inc. (Akita, Japan).

The primary efficacy endpoint was the percent change in fasting serum TG from baseline to the last evaluation point. The primary safety endpoints were the incidence of an AE or ADR occurring after drug administration during the study. Secondary efficacy endpoints included percent changes in lipid variables and changes in inflammation variables at week 52 [using the last-observation-carried-forward (LOCF)]. Secondary safety endpoints included changes in the levels of aspartate aminotransferase (AST), ALT, $\gamma$-GT, ALP, serum creatinine, eGFR, and CK at week 52. Each baseline value was defined as (1) the mean of the corresponding values in the first and second tests at the screening examination and at week 0 of the treatment period for fasting serum TG, HDL-C, total cholesterol (TC), LDL-C, and non-HDL-C; and (2) the value at week 0 of the treatment period for the other secondary variables. Efficacy and safety were established post hoc by subgroups stratified by baseline eGFR as follows: G1 (normal or high; $\geq 90 \mathrm{~mL} / \mathrm{min} / 1.73 \mathrm{~m}^{2}$ ), G2 (mildly decreased; $\geq 60$ and $<90 \mathrm{~mL} / \mathrm{min} / 1.73 \mathrm{~m}^{2}$ ), G3a-G3b (mildly to moderately decreased and moderately to severely decreased; $\geq 30$ and $<60 \mathrm{~mL} / \mathrm{min} / 1.73 \mathrm{~m}^{2}$ ), and G4-G5 (severely decreased and kidney failure; $<30 \mathrm{~mL} / \mathrm{min} / 1.73 \mathrm{~m}^{2}$ ), according to the Kidney Disease Improving Global Outcomes (KDIGO) 2012 Clinical Practice Guideline for the Evaluation and Management of Chronic Kidney Disease [48]. The plasma concentration of pemafibrate was measured with liquid chromatography-tandem mass spectrometry (LC-MS-MS) only at the institutions where this procedure was feasible and in patients who provided informed consent. Blood sampling for trough values was conducted before the morning dose of pemafibrate in parallel with that for fasting blood laboratory tests in weeks 4, 8, and 12 of the treatment period. Blood sampling after pemafibrate administration was carried out once between weeks 4 and 24 at $0.5-1.5,1.5-3$, and $4-6 \mathrm{~h}$ after pemafibrate administration.

The target sample size was set to 170 patients based on the number required to evaluate safety according to the International Conference on Harmonisation (ICH)-E1 guideline "Extent of population exposure to assess clinical safety for drugs intended for long-term treatment of non-life-threatening conditions". For the primary efficacy endpoint, one-sample $t$-tests were performed. The numbers of patients with AEs and ADRs and the incidences of AEs and ADRs were calculated in the analysis of primary safety endpoints. For the secondary efficacy endpoints, one-sample $t$-tests or Wilcoxon signed-rank tests [for high-sensitivity C-reactive protein (hsCRP) and IL-1 $\beta$ ] were performed. The secondary safety endpoints were analyzed with Wilcoxon signed-rank tests. A two-sided significance level of 0.05 was used. SAS v. 9.3 (SAS Institute Inc., Cary, NC, USA) was used for these analyses. Where indicated, the data are expressed as means \pm standard deviation (SD).

\section{Conclusions}

In conclusion, 0.2 to $0.4 \mathrm{mg} /$ day pemafibrate presented a good safety profile and excellent efficacy to treat serum lipid abnormalities in a broad range of patients, including those with CKD.

Author Contributions: K.Y., S.Y., H.A., E.A., H.S., and S.I. contributed to the concept, design, and execution of the study, and to the interpretation of the data. K.Y. contributed substantially to the writing and critical review of the manuscript. S.Y., H.A., E.A., and S.I. contributed to the critical review of the manuscript. H.S. is the guarantor of this work and, as such, had full access to all of the data in the study and takes responsibility for the data integrity and accuracy of the data analysis.

Funding: This study was funded by Kowa Company, Ltd. The study sponsor had a role in the study design; data collection, analysis, and interpretation; and writing of the report.

Acknowledgments: The authors acknowledge the investigators and patients who participated in this study. This study was conducted at Yamagata Tokushukai Hospital (Isoji Sasagawa), Koga General Hospital (Masataka Fukue), 
Joumou Ohashi Clinic (Tetsuya Shigehara), Maebashi Hirosegawa Clinic (Shintaro Yano), Sakado Central Hospital (Nobuki Takata), Kanauchi Medical Clinic (Naohisa Hoshino), Shinkoiwa Ekimae Sougou Clinic (Chiaki Noguchi), Tokyo-Eki Center-building Clinic (Arihiro Kiyosue), IMSUT Hospital, The Institute of Medical Science, The University of Tokyo (Naoya Kato), Meiwa Hospital (Kazuyuki Mizuyama), St. Marianna University School of Medicine (Yoshio Nagai), Yokosuka Kyosai Hospital (Teiichi Tamura), Yokohama Shin-midori General Hospital (Takashi Kirita), Shinrakuen Hospital (Shigeru Miyazaki), Japanese Red Cross Society Suwa Hospital (Naoki Tachibana), Chubu Rosai Hospital (Eitaro Nakashima), Nagoya Kyoritsu Hospital (Hirotake Kasuga), Fujita Health University Hospital (Yukio Yuzawa), Uji-Tokushukai Medical Center (Atsushi Sueyoshi), Kyoto University Hospital (Nobuya Inagaki), Horikawa Hospital (Yasumasa Kondo), OCROM Clinic (Satoshi Inoue), Osaka Pref. Saiseikai Tondabayashi Hospital (Takeshi Kubota), Kishiwada Tokushukai Hospital (Shinichi Higashiue), Matsue Red Cross Hospital (Toshiaki Sato), JA Hiroshima General Hospital (Shuji Tsujiyama), Hiroshima City Hiroshima Citizens Hospital (Shoichiro Kojo), Mazda Hospital (Kotaro Sumii), Wellness Tenjin Clinic (Toshihiko Hashimoto), Nakamura Cardiovascular Clinic (Yuichiro Nakamura), SOUSEIKAI PS Clinic (Masanari Shiramoto), and Fukuoka University Hospital (Hitoshi Nakashima). The authors would like to thank Wolters Kluwer and Editage (www.editage.com) for English language editing.

Conflicts of Interest: K.Y. reports personal fees from Kowa during the conduct of the study; personal fees from Astellas Pharma, Amgen Astellas BioPharma, AstraZeneca, Bayer, MSD, Ono Pharmaceutical, Sanwa Kagaku Kenkyusho, Kissei Pharmaceutical, Kyowa Hakko Kirin, Kaken Pharmaceutical, Kowa, Kowa Pharmaceutical, Sanofi, Shionogi, Daiichi Sankyo, Taisho Toyama Pharmaceutical, Sumitomo Dainippon Pharma, Takeda Pharmaceutical, Mitsubishi Tanabe Pharma, Eli Lilly Japan, Nippon Boehringer Ingelheim, Novartis Pharma, Novo Nordisk Pharma, Pfizer Japan, Mochida Pharmaceutical, and Teijin Pharma; and grants from Astellas Pharma, MSD, Ono Pharmaceutical, Kowa Pharmaceutical, Kyowa Hakko Kirin, Shionogi, Daiichi Sankyo, Taisho Toyama Pharmaceutical, Sumitomo Dainippon Pharma, Takeda Pharmaceutical, Mitsubishi Tanabe Pharma, Teijin Pharma, Eli Lilly Japan, Nippon Boehringer Ingelheim, Pfizer Japan, Novo Nordisk Pharma, and Mochida Pharmaceutical outside of the submitted work. S.Y. reports personal fees from Kowa during the conduct of the study; personal fees from Kowa, MSD, Amgen Astellas BioPharma, and Sanofi; and grants from MSD, Bayer Yakuhin, Nippon Boehringer Ingelheim, Takeda Pharmaceutical, Ono Pharmaceutical, Astellas Pharma, Mitsubishi Tanabe Pharma, Kyowa Medex, and Rohto Pharmaceutical outside of the submitted work. H.A. reports personal fees from Kowa during the conduct of the study; personal fees from Daiichi Sankyo, Astellas Pharma, Sanofi, Kowa, MSD, Amgen Astellas BioPharma, and Abbott Japan; and grants from Daiichi Sankyo outside of the submitted work. E.A. reports personal fees from Kowa during the conduct of the study; personal fees from Astellas Pharma, MSD, Sanofi, Ono Pharmaceutical, and Novo Nordisk Pharma; and grants from Astellas Pharma, Ono Pharmaceutical, Nippon Boehringer Ingelheim, Taisho Toyama Pharmaceutical, Daiichi Sankyo, MSD, Sanofi, Takeda Pharmaceutical, Mitsubishi Tanabe Pharma, Novartis Pharma, Novo Nordisk Pharma, and Eli Lilly Japan outside of the submitted work. H.S. is an employee of Kowa Company, Ltd. S.I. reports personal fees from Kowa during the conduct of the study; personal fees from MSD, Sanofi, Amgen Astellas BioPharma, Kowa Pharmaceutical, and Kowa; and grants from Eli Lilly Japan, Sanofi, Astellas Pharma, Nippon Boehringer Ingelheim, Mitsubishi Tanabe Pharma, Daiichi Sankyo, Takeda Pharmaceutical, Ono Pharmaceutical, Shionogi, Teijin Pharma, and Kowa Pharmaceutical outside of the submitted work.

\section{Abbreviations}

$\begin{array}{ll}\text { ADR } & \text { Adverse drug reaction } \\ \text { AE } & \text { Adverse event } \\ \text { ALP } & \text { Akaline phosphatase } \\ \text { ALT } & \text { Alanine aminotransferase } \\ \text { Apo } & \text { Apolipoprotein } \\ \text { ASCVD } & \text { Atherosclerotic cardiovascular disease } \\ \text { AST } & \text { Aspartate aminotransferase } \\ \text { CK } & \text { Creatine kinase } \\ \text { CKD } & \text { Chronic kidney disease } \\ \text { CM } & \text { Chylomicron } \\ \text { CM-C } & \text { CM-cholesterol } \\ \text { CVD } & \text { Cardiovascular disease } \\ \text { eGFR } & \text { Estimated glomerular filtration rate } \\ \text { GCP } & \text { Good Clinical Practice } \\ \text { HbA1c } & \text { Hemoglobin A1c } \\ \text { HDL } & \text { High-density lipoprotein } \\ \text { HDL-C } & \text { HDL cholesterol } \\ \text { HPLC } & \text { High-performance liquid chromatography } \\ \text { hsCRP } & \text { High-sensitivity C-reactive protein }\end{array}$




$\begin{array}{ll}\text { ICH } & \text { International Conference on Harmonisation } \\ \text { IL-1 } \beta & \text { Interleukin-1 } \beta \\ \text { JAPIC } & \text { Japan Pharmaceutical Information Center } \\ \text { KDIGO } & \text { Kidney Disease Improving Global Outcomes } \\ \text { LCAT } & \text { Lecithin-cholesterol acyltransferase } \\ \text { LC-MS-MS } & \text { Liquid chromatography-tandem mass spectrometry } \\ \text { LDL } & \text { Low-density lipoprotein } \\ \text { LDL-C } & \text { LDL cholesterol } \\ \text { LOCF } & \text { Last-observation-carried-forward } \\ \text { LPL } & \text { Lipoprotein lipase } \\ \text { PPAR } \alpha & \text { Peroxisome proliferator-activated receptor } \alpha \\ \text { RemL-C } & \text { Remnant lipoprotein cholesterol } \\ \text { SAE } & \text { Serious adverse event } \\ \text { SD } & \text { Standard deviation } \\ \text { SPPARM } \alpha & \text { Selective peroxisome proliferator-activated receptor- } \alpha \text { modulator } \\ \text { TC } & \text { Total cholesterol } \\ \text { TG } & \text { Triglyceride } \\ \text { ULN } & \text { Upper limit of normal } \\ \text { VLDL } & \text { Very-low-density lipoprotein } \\ \text { VLDL-C } & \text { VLDL-cholesterol } \\ \gamma \text {-GT } & \gamma \text {-Glutamyltransferase } \\ & \end{array}$

\section{References}

1. Knopp, R.H. Drug treatment of lipid disorders. N. Engl. J. Med. 1999, 341, 498-511. [CrossRef] [PubMed]

2. Murray, C.J.; Lopez, A.D. Mortality by cause for eight regions of the world: Global Burden of Disease Study. Lancet 1997, 349, 1269-1276. [CrossRef]

3. Fruchart, J.C.; Sacks, F.M.; Hermans, M.P.; Assmann, G.; Brown, W.V.; Ceska, R.; Chapman, M.J.; Dodson, P.M.; Fioretto, P.; Ginsberg, H.N.; et al. The Residual Risk Reduction Initiative: A call to action to reduce residual vascular risk in dyslipidaemic patient. Diabetes Vasc. Dis. Res. 2008, 5, 319-335. [CrossRef] [PubMed]

4. Chapman, M.J.; Redfern, J.S.; McGovern, M.E.; Giral, P. Niacin and fibrates in atherogenic dyslipidemia: Pharmacotherapy to reduce cardiovascular risk. Pharmacol. Ther. 2010, 126, 314-345. [CrossRef] [PubMed]

5. Matsushita, K.; van der Velde, M.; Astor, B.C.; Woodward, M.; Levey, A.S.; de Jong, P.E.; Coresh, J.; Gansevoort, R.T. Association of estimated glomerular filtration rate and albuminuria with all-cause and cardiovascular mortality in general population cohorts: A collaborative meta-analysis. Lancet 2010, 375, 2073-2081. [CrossRef] [PubMed]

6. Levey, A.S.; de Jong, P.E.; Coresh, J.; El Nahas, M.; Astor, B.C.; Matsushita, K.; Gansevoort, R.T.; Kasiske, B.L.; Eckardt, K.U. The definition, classification, and prognosis of chronic kidney disease: A KDIGO Controversies Conference report. Kidney Int. 2011, 80, 17-28. [CrossRef] [PubMed]

7. Vaziri, N.D. Dyslipidemia of chronic renal failure: The nature, mechanisms, and potential consequences. Am. J. Physiol. Ren. Physiol. 2006, 290, F262-F272. [CrossRef] [PubMed]

8. Okopien, B.; Buldak, L.; Boldys, A. Fibrates in the management of atherogenic dyslipidemia. Expert Rev. Cardiovasc. Ther. 2017, 15, 913-921. [CrossRef] [PubMed]

9. Williams, A.J.; Baker, F.; Walls, J. The short term effects of bezafibrate on the hypertriglyceridaemia of moderate to severe uraemia. Br. J. Clin. Pharmacol. 1984, 18, 361-367. [CrossRef] [PubMed]

10. Lipscombe, J.; Lewis, G.F.; Cattran, D.; Bargman, J.M. Deterioration in renal function associated with fibrate therapy. Clin. Nephrol. 2001, 55, 39-44. [PubMed]

11. Broeders, N.; Knoop, C.; Antoine, M.; Tielemans, C.; Abramowicz, D. Fibrate-induced increase in blood urea and creatinine: Is gemfibrozil the only innocuous agent? Nephrol. Dial. Transplant. 2000, 15, 1993-1999. [CrossRef] [PubMed]

12. McQuade, C.R.; Griego, J.; Anderson, J.; Pai, A.B. Elevated serum creatinine levels associated with fenofibrate therapy. Am. J. Health Syst. Pharm. 2008, 65, 138-141. [CrossRef] [PubMed] 
13. Ritter, J.L.; Nabulsi, S. Fenofibrate-Induced elevation in serum creatinine. Pharmacotherapy 2001, 21, 1145-1149. [CrossRef] [PubMed]

14. Mottl, A.K.; Buse, J.B.; Ismail-Beigi, F.; Sigal, R.J.; Pedley, C.F.; Papademetriou, V.; Simmons, D.L.; Katz, L.; Mychaleckyj, J.C.; Craven, T.E. Long-Term Effects of Intensive Glycemic and Blood Pressure Control and Fenofibrate Use on Kidney Outcomes. Clin. J. Am. Soc. Nephrol. 2018, 13, 1693-1702. [CrossRef] [PubMed]

15. Davidson, M.H.; Armani, A.; McKenney, J.M.; Jacobson, T.A. Safety considerations with fibrate therapy. Am. J. Cardiol. 2007, 99, 3c-18c. [CrossRef] [PubMed]

16. Kostapanos, M.S.; Florentin, M.; Elisaf, M.S. Fenofibrate and the kidney: An overview. Eur. J. Clin. Investig. 2013, 43, 522-531. [CrossRef] [PubMed]

17. Attridge, R.L.; Frei, C.R.; Ryan, L.; Koeller, J.; Linn, W.D. Fenofibrate-associated nephrotoxicity: A review of current evidence. Am. J. Health Syst. Pharm. 2013, 70, 1219-1225. [CrossRef] [PubMed]

18. Catapano, A.L.; Graham, I.; De Backer, G.; Wiklund, O.; Chapman, M.J.; Drexel, H.; Hoes, A.W.; Jennings, C.S.; Landmesser, U.; Pedersen, T.R.; et al. 2016 ESC/EAS Guidelines for the Management of Dyslipidaemias: The Task Force for the Management of Dyslipidaemias of the European Society of Cardiology (ESC) and European Atherosclerosis Society (EAS) Developed with the special contribution of the European Assocciation for Cardiovascular Prevention \& Rehabilitation (EACPR). Atherosclerosis 2016, 253, 281-344. [CrossRef]

19. Catapano, A.L.; Graham, I.; De Backer, G.; Wiklund, O.; Chapman, M.J.; Drexel, H.; Hoes, A.W.; Jennings, C.S.; Landmesser, U.; Pedersen, T.R.; et al. 2016 ESC/EAS Guidelines for the Management of Dyslipidaemias. Eur. Heart J. 2016, 37, 2999-3058. [CrossRef]

20. Graham, D.J.; Staffa, J.A.; Shatin, D.; Andrade, S.E.; Schech, S.D.; La Grenade, L.; Gurwitz, J.H.; Chan, K.A.; Goodman, M.J.; Platt, R. Incidence of hospitalized rhabdomyolysis in patients treated with lipid-lowering drugs. JAMA 2004, 292, 2585-2590. [CrossRef]

21. Yamazaki, Y.; Abe, K.; Toma, T.; Nishikawa, M.; Ozawa, H.; Okuda, A.; Araki, T.; Oda, S.; Inoue, K.; Shibuya, K.; et al. Design and synthesis of highly potent and selective human peroxisome proliferator-activated receptor alpha agonists. Bioorg. Med. Chem. Lett. 2007, 17, 4689-4693. [CrossRef] [PubMed]

22. Ishibashi, S.; Yamashita, S.; Arai, H.; Araki, E.; Yokote, K.; Suganami, H.; Fruchart, J.C.; Kodama, T. Effects of K-877, a novel selective PPARalpha modulator (SPPARMalpha), in dyslipidaemic patients: A randomized, double blind, active- and placebo-controlled, phase 2 trial. Atherosclerosis 2016, 249, 36-43. [CrossRef]

23. Arai, H.; Yamashita, S.; Yokote, K.; Araki, E.; Suganami, H.; Ishibashi, S. Efficacy and safety of K-877, a novel selective peroxisome proliferator-activated receptor alpha modulator (SPPARMalpha), in combination with statin treatment: Two randomised, double-blind, placebo-controlled clinical trials in patients with dyslipidaemia. Atherosclerosis 2017, 261, 144-152. [CrossRef] [PubMed]

24. Ishibashi, S.; Arai, H.; Yokote, K.; Araki, E.; Suganami, H.; Yamashita, S. Efficacy and safety of pemafibrate (K-877), a selective peroxisome proliferator-activated receptor alpha modulator, in patients with dyslipidemia: Results from a 24-week, randomized, double blind, active-controlled, phase 3 trial. J. Clin. Lipidol. 2018, 12, 173-184. [CrossRef] [PubMed]

25. Araki, E.; Yamashita, S.; Arai, H.; Yokote, K.; Satoh, J.; Inoguchi, T.; Nakamura, J.; Maegawa, H.; Yoshioka, N.; Tanizawa, Y.; et al. Effects of Pemafibrate, a Novel Selective PPARalpha Modulator, on Lipid and Glucose Metabolism in Patients With Type 2 Diabetes and Hypertriglyceridemia: A Randomized, Double-Blind, Placebo-Controlled, Phase 3 Trial. Diabetes Care 2018, 41, 538-546. [CrossRef] [PubMed]

26. Arai, H.; Yamashita, S.; Yokote, K.; Araki, E.; Suganami, H.; Ishibashi, S. Efficacy and Safety of Pemafibrate Versus Fenofibrate in Patients with High Triglyceride and Low HDL Cholesterol Levels: A Multicenter, Placebo-Controlled, Double-Blind, Randomized Trial. J. Atheroscler. Thromb. 2018, 25, 521-538. [CrossRef] [PubMed]

27. Blair, H.A. Pemafibrate: First Global Approval. Drugs 2017, 77, 1805-1810. [CrossRef] [PubMed]

28. Fruchart, J.C. Selective peroxisome proliferator-activated receptor alpha modulators (SPPARMalpha): The next generation of peroxisome proliferator-activated receptor alpha-agonists. Cardiovasc. Diabetol. 2013, 12, 82. [CrossRef] [PubMed]

29. Fruchart, J.C. Pemafibrate (K-877), a novel selective peroxisome proliferator-activated receptor alpha modulator for management of atherogenic dyslipidaemia. Cardiovasc. Diabetol. 2017, 16, 124. [CrossRef] [PubMed] 
30. Hounslow, N.; Mair, S.; Suganami, H.; Nakamura, M. Pemafibrate Has High Bioavailability and is Principally Excreted via the Liver. Atheroscler. Suppl. 2018, 32, 157. [CrossRef]

31. Hosford, D.; Gordon, G.; Suganami, H.; Nakamura, M. The Plasma Concentration and Pharmacokinetic Parameters of Pemafibrate Did Not Increase in a Creatinine Clearance-Dependent Manner. Atheroscler. Suppl. 2018, 32, 150. [CrossRef]

32. Reiss, A.B.; Voloshyna, I.; De Leon, J.; Miyawaki, N.; Mattana, J. Cholesterol Metabolism in CKD. Am. J. Kidney Dis. 2015, 66, 1071-1082. [CrossRef] [PubMed]

33. Molitch, M.E. Management of dyslipidemias in patients with diabetes and chronic kidney disease. Clin. J. Am. Soc. Nephrol. 2006, 1, 1090-1099. [CrossRef] [PubMed]

34. Tsimihodimos, V.; Mitrogianni, Z.; Elisaf, M. Dyslipidemia associated with chronic kidney disease. Open Cardiovasc. Med. J. 2011, 5, 41-48. [CrossRef] [PubMed]

35. Kaysen, G.A. Lipid-Lowering Therapy in CKD: Should We Use It and in Which Patients. Blood Purif. 2017, 43, 196-199. [CrossRef] [PubMed]

36. Honda, H.; Hirano, T.; Ueda, M.; Kojima, S.; Mashiba, S.; Hayase, Y.; Michihata, T.; Shibata, T. High-Density Lipoprotein Subfractions and Their Oxidized Subfraction Particles in Patients with Chronic Kidney Disease. J. Atheroscler. Thromb. 2016, 23, 81-94. [CrossRef] [PubMed]

37. Remick, J.; Weintraub, H.; Setton, R.; Offenbacher, J.; Fisher, E.; Schwartzbard, A. Fibrate therapy: An update. Cardiol. Rev. 2008, 16, 129-141. [CrossRef]

38. Liang, C.C.; Wang, I.K.; Kuo, H.L.; Yeh, H.C.; Lin, H.H.; Liu, Y.L.; Hsu, W.M.; Huang, C.C.; Chang, C.T. Long-term use of fenofibrate is associated with increased prevalence of gallstone disease among patients undergoing maintenance hemodialysis. Ren. Fail. 2011, 33, 489-493. [CrossRef]

39. Makowka, A.; Dryja, P.; Chwatko, G.; Bald, E.; Nowicki, M. Treatment of chronic hemodialysis patients with low-dose fenofibrate effectively reduces plasma lipids and affects plasma redox status. Lipids Health Dis. 2012, 11, 47. [CrossRef]

40. Abdel Magid, A.M.; Abbassi, M.M.; Iskander, E.E.M.; Mohamady, O.; Farid, S.F. Randomized comparative efficacy and safety study of intermittent simvastatin versus fenofibrate in hemodialysis. J. Comp. Eff. Res. 2017, 6, 413-424. [CrossRef]

41. FDA Approved Drug Products, AbbVie Inc. TRILIPIX (Fenofibric Acid) Capsule [Package Insert]. North Chicago, IL. 2018. Available online: https:/ / www.accessdata.fda.gov/scripts/cder/daf/index.cfm?event= overview.process\&applno=022224 (accessed on 1 February 2019).

42. Desager, J.P.; Costermans, J.; Verberckmoes, R.; Harvengt, C. Effect of hemodialysis on plasma kinetics of fenofibrate in chronic renal failure. Nephron 1982, 31, 51-54. [CrossRef] [PubMed]

43. MHRA, Actavis Group PTC ehf. BEZALIP [Summary of Product Characteristics]. Iceland. September 2016. Available online: http:/ / www.mhra.gov.uk/spc-pil/?prodName=BEZALIP\&subsName=\&pageID= ThirdLevel\&searchTerm=Bezalip\#retainDisplay (accessed on 1 February 2019).

44. Abshagen, U.; Kosters, W.; Kaufmann, B.; Lang, P.D. Pharmacokinetics of bezafibrate after single and multiple doses in the presence of renal failure. Klin. Wochenschr. 1980, 58, 889-896. [CrossRef] [PubMed]

45. Anderson, P.; Norbeck, H.E. Clinical pharmacokinetics of bezafibrate in patients with impaired renal function. Eur. J. Clin. Pharmacol. 1981, 21, 209-214. [CrossRef]

46. FDA Approved Drug Products, Parke-Davis, Division of Pfizer Inc. LOPID (Gemfibrozil Tables, USP) [Package Insert]. April 2018. Available online: https:/ /www.accessdata.fda.gov/scripts/cder/daf/index. cfm?event=overview.process\&applno=018422 (accessed on 1 February 2019).

47. Furusyo, N.; Ai, M.; Okazaki, M.; Ikezaki, H.; Ihara, T.; Hayashi, T.; Hiramine, S.; Ura, K.; Kohzuma, T.; Schaefer, E.J.; et al. Serum cholesterol and triglyceride reference ranges of twenty lipoprotein subclasses for healthy Japanese men and women. Atherosclerosis 2013, 231, 238-245. [CrossRef] [PubMed]

48. KDIGO 2012 Clinical Practice Guideline for the Evaluation and Management of Chronic Kidney Disease. Kidney. Int. Suppl. 2013, 3, 5-14. [CrossRef]

(C) 2019 by the authors. Licensee MDPI, Basel, Switzerland. This article is an open access article distributed under the terms and conditions of the Creative Commons Attribution (CC BY) license (http:/ / creativecommons.org/licenses/by/4.0/). 